\title{
Plant species and products of the Traditional Chinese Phytotherapy in the Ciudad Autónoma de Buenos Aires, Argentina
}

\author{
Julio Alberto Hurrell ${ }^{1 *}$ and Jeremías P. Puentes ${ }^{1}$
}

\begin{abstract}
This paper is focused in medicinal plant species belonging to the Traditional Chinese Phytotherapy whose products are commercialized in the pluricultural context of the Ciudad Autónoma de Buenos Aires, Argentina. The Chinese immigrants segment, like other recent immigrants groups in the study area, introduce their own plant products into the local urban scenario. In this framework, not only enter the new products but also their knowledge and beliefs associated, that become part of the mosaic of the knowledge in the local pluricultural system. Thus, the urban botanical knowledge constitutes a complex that includes the knowledge linked to the origin traditions of the immigrants segments, and the nontraditional one: the knowledge taught and learned, and the transmitted by the media, including scientific knowledge. The plant products incorporated by Chinese immigrants to their own restricted commercial circuit (linked to their traditions) are invisible for the majority of the local inhabitants. However, some of these products enter the general commercial circuit (nontraditional) and become visible for all local population. This visualization of the plant products implies the transmission of its associated knowledge, a process enhanced by the mass media, mainly the Internet. The results are interpreted within this theoretical framework. Also, the main features of the Traditional Chinese Phytotherapy are summarized, due to its differences with our Western medicine. For the species considered the scientific names, botanical families, distribution, Chinese name and Latin denomination of the plant products, and the reference samples are indicated. For each species are included a list of its uses linked to traditions, and a revision work of the biological activity and effects evaluated (the validation context in terms of Western science). In the context of Urban Ethnobotany, the presence alone of invisible species is a significant issue because increases the local biocultural diversity (of useful plants, products, and its associated knowledge). Likewise, the species that become visible make evident the dynamics of changes in their visualization process, an adaptive phenomenon that allow the understanding of the local biocultural system complexity.
\end{abstract}

Keywords: Urban Ethnobotany - Urban Pluriculturality - Botanical knowledge complexity Traditional Chinese Medicine, Buenos Aires city

1 - Laboratorio de Etnobotánica y Botánica Aplicada (LEBA), Facultad de Ciencias Naturales y Museo, Universidad Nacional de La Plata. Calle 64 nro. 3, 1900-La Plata. Consejo Nacional de Investigaciones Científicas y Técnicas (CONICET), República Argentina

*Corresponding author: juliohurrell@gmail.com 


\section{INTRODUCTION}

This contribution presents the results of an ethnobotanical survey on plants of Traditional Chinese Phytotherapy introduced by Chinese immigrants in the pluricultural context of the Ciudad Autónoma de Buenos Aires, Argentina, and its traditional and biomedical uses. Ethnobotany is understood here in a broad sense, as the study of the complexity of the relationships between people and plants (Albuquerque and Hurrell 2010). In this framework, Urban Ethnobotany can be defined as the Ethnobotany of the pluricultural contexts that constitute the urban areas (Hurrell 2014a). Urban pluriculturality (heterogeneity) is enriched by the increasing presence of diverse immigrants segments (immigration both longstanding and recent) which introduce the plants and its associated knowledge into the local scenario.

The Botanical Knowledge (BK) constitutes a complex set of knowledge and beliefs about the plant environment elements: plants, parts thereof, derivative products. In urban areas, BK includes (at least) two subsets that interact in different ways (does not always clear): 1) "nontraditional", e.g. the knowledge that are taught and learned, and the transmitted by the media, including the scientific one, 2) "linked to traditions", e.g. local longstanding family traditions, and origin traditions of the immigrants segments (Hurrell and Pochettino 2014; Hurrell et al. 2015a, 2015b; Hurrell 2014a). The latter subset cannot be considered as Traditional Botanical Knowledge (TBK), in a narrow sense, because the TBK mainly corresponds to more homogeneous cultural contexts. Also, to assume that all the urban BK (UBK) is a nontraditional knowledge would be fall into a reductionism, because we would be hiding the local cultural heterogeneity, i.e. the base of its complexity.

This contribution is framed in a research line on Urban Ethnobotany of the Laboratorio de Etnobotánica y Botánica Aplicada (LEBA), developed for more than a decade in the Área Metropolitana Buenos Aires-La Plata (Hurrell and Pochettino 2014). This framework argues that the UBK is not accessible in a direct way, but can be extrapolated from the actions that this knowledge orients, e.g. strategies of selection, use, and consume of plants or its products. At the same time, these actions become evident through the circulation of plant products inside the local commercial circuits. This methodological proposal involves evaluate: 1) the diffusion of plant products, 2) the action strategies that allow such dissemination, 3) the UBK that guides the whole process (Hurrell 2014a). These theoretical premises are consistent with the distinction established by Ladio and Albuquerque for Urban Ethnobiology, between "tangible" components, i.e. the biological resources (in our context, circulating plant products) and "intangible" components: values, norms, and rules prevailing in each particular worldview, which we identify with the local UBK (Ladio and Albuquerque 2014, 2016).

Plant products circulating inside the general commercial circuit are "visible" to all urban inhabitants (including the different immigrants segments). Plant products circulating inside restricted commercial circuit of each immigrants segment are visible to the members of that particular segment and some urban inhabitants (nonimmigrants and immigrants from other segments) interested in specific plant products. However, in general terms, the products that are exclusive of the commercial circuit of a particular immigrants 
segment are "invisible" for the majority of the inhabitants of the local pluricultural context. At times, some invisible plant products (tangible components) and its knowledge linked to traditions (intangible components) become visible when entering the general commercial circuit (nontraditional scenario). In these situations, a "visualization process" occurs: invisible plant products gain visibility (examples of plant species illustrating this process are presented in Results and Discussion). This is a process enhanced by the mass media, especially the Internet that plays a key role in the knowledge transmission in a rapid way and in multiple directions at the same time (Hurrell 2014a). The visualization process implies a contextual change, in which the knowledge linked to the immigrants' traditions becomes a nontraditional knowledge. In this sense, the distinction between traditional and nontraditional components is "complex and often transient", as had been suggested by Ladio and Albuquerque (2016).

This paper focuses on the segment of Chinese immigrants. In the first half of the twentieth century the Chinese immigration was low and settled in the Buenos Aires Metropolitan Area, but since the late twentieth century, Chinese immigrants outnumbered other groups of Asian immigrants, such as the Japanese and Koreans, who previously dominated. In Argentina, Chinese segment comprising about 120,000 immigrants. It is the fourth largest group of recent immigrants after those from Bolivia, Paraguay, and Peru (Bogado-Bordazar 2003; Cerrutti 2009; Hurrell and Pochettino 2014; Marcos and Mera 2015; Sassone and Mera 2007).

The Traditional Chinese Medicine (TCM) includes a set of therapeutic practices produced from experiences for more than three millennia. TCM and the Ayurveda, the
Traditional Indian Medicine, remain the most ancient yet living traditions from the East (Patwardhan et al. 2005). Both traditions have been spread in the West, though its principles are complex and not always well understood. The main treatment modality of the TCM is the Traditional Chinese Herbal Medicine or Traditional Chinese Phytotherapy (TCP) that is based on herbal products from different plant species, and mainly its combinations. It is founded on clinical practices within an integrative approach: "Therapy is based on understanding relations between part and whole, distinguishing symptom and appearance from true cause, and treating each individual case as unique" (Wu 2005).

Chinese herbal theory assumes that the body is traversed by the vital energy, called Qi (or Chi), which regulates the physical, mental, emotional and spiritual balance. This energy is affected by the opposing forces of Yin and Yang (universal dual principle), and the disease arises from altering the vital energy flow, when an imbalance of Yin-Yang occurs. The Qi circulates through the body by specific channels named "meridians", that connecting different organs. The Chinese organ system or Zang-fu is based more on organs functions than in their anatomy, e.g., the heart meridian is linked with their specific functions and also conditions like anxiety and/or restlessness. The meridians related to organs include: heart, lung, spleen, gallbladder, liver, kidney, bladder, stomach, small intestine, large intestine. Herbal remedies can be characterized by those meridians, and also by its properties: "temperature" and "flavor". Temperature includes: hot, warm, neutral, cool, and cold, e.g., "hot and warm herbs" can be useful to treat "cool or cold diseases", and vice versa. Flavor includes: acrid, bitter, sweet, sour, and salty, it is said that bitter herbs are used 
to drain (e.g. purges the bowels) and dry (e.g. in cases as leukorrhea or diarrhea), while the sweet herbs nourish and tonify, e.g., the Qi (Adams and Lien 2013; Leung et al. 2003; Wu 2005; Xutian et al. 2014; Zhu 1998).

\section{MATERIAL AND METHODS}

\section{Study area}

The study area corresponds to the Ciudad Autónoma de Buenos Aires, federal capital of Argentina. It has $202 \mathrm{~km}^{2}$ and $2,890,000$ inhabitants, according to the National Census 2010 (INDEC 2016); the population density is about 14,300 inhabitants / $\mathrm{km}^{2}$. This city and 24 surrounding districts of Buenos Aires province constitute the Buenos Aires Metropolitan Area, with $2081 \mathrm{~km}^{2}$ and about $15,000,000$ inhabitants (as of 2014). The population density is about 7200 inhabitants $/ \mathrm{km}^{2}$. This metropolitan area is the largest in the country in both size and population, the second in South America (after the metropolitan area of São Paulo, Brazil), the third in Latin America (after São Paulo and Mexico DF.), the fifth of America and the seventeenth in the world (Forstall et al. 2009; Hurrell and Pochettino 2014).

\section{Actors involved}

Argentina has received massive waves of immigration from the midnineteenth century and first half of the twentieth century. Most of these immigrants were of European origin: $44.9 \%$ Italian and $31.5 \%$ Spaniards on the total of immigrants registered until 1940. These migration flows have helped to shape the country's cultural heritage, and many current "family traditions" have their roots in that early immigration. In the second half of the twentieth century, a new kind of recent immigration occurred, not massive, mainly from neighboring countries, whose destiny focused in Buenos Aires Metropolitan Area. Another recent immigration in the area corresponds to Far Eastern countries such as Korea, Japan, and China. Asian immigration in 2001 represented almost $2 \%$ of all foreigners in the country, meager value compared to $67.96 \%$ coming from American countries and $28.22 \%$ from European countries (BogadoBordazar 2003; Cerrutti 2009; Hurrell and Pochettino 2014; Marcos and Mera 2015; Sassone and Mera 2007). In this context, the research line of the LEBA works with three segments of recent immigrants taken as reference: Bolivian, Paraguayan, and Chinese. For the Bolivian segment, partial results have been presented previously, in which different plant products in visualization process were identified (Pochettino et al. 2012; Puentes and Hurrell 2015). For the Paraguayan segment a preliminary work were recently presented (Hurrell et al. 2016). This time results of ongoing studies for the Chinese immigrants segment are presented. These results are circumscribed to medicinal products belonging to 32 plant species of the Traditional Chinese Phytotherapy. In previous scientific meetings 12 species (Hurrell 2014b) and 10 species (Hurrell and Puentes 2015) were cited. For this paper, the information about the above mentioned 22 species is updated, and 10 new species are added.

Of the total of Chinese immigrants in Argentina, it is estimated that $80 \%$ live in Buenos Aires city. The most visible presence of this segment is located in a sector of Belgrano neighborhood, called "Barrio Chino", where restaurants, supermarkets and shops were installed, and various cultural events related to Chinese festivities 
are organized. These characteristics replicate the profile of Chinatowns in other large metropolitan areas of the world, like San Francisco, Paris, Sydney, and Toronto, among the main (Sassone and Mera 2007). The supermarkets in the Barrio Chino offer products that satisfy the demands of the community itself, other communities (Japanese, Korean, Arabic), and local residents looking for new specific products. It is estimated that the Barrio Chino receives about 15,000 visitors every weekend. For the Chinese segment it embodies a space for local tourism: restaurants offering Chinese foods oriented to "Argentine taste". Paradoxically, restaurants that offer dishes preferred by the Chinese immigrants are not located inside the Barrio Chino (BogadoBordazar 2003). Nevertheless, mentioned supermarkets introduce a wide variety of novel plant products and constitute true centers for dissemination of products (and its associated botanical knowledge) for local residents. The plant products that sell these supermarkets become visible when enter the general commercial circuit.

\section{Field works}

The ethnobotanical survey was focused on five large supermarkets of Barrio Chino, to analyze the restricted commercial circuit of Chinese immigrants (linked to their origin traditions), and 120 health food stores (locally called dietéticas) of the general commercial circuit, in order to evaluate the visibility of plant species and products in the urban local scenario. The surveys followed usual qualitative methods and techniques: participant observation, free listings, semistructured and free interviews (Albuquerque et al. 2014; Etkin and Ticktin 2010; Quinlan 2005; Stepp 2005). With the prior informed consent, 250 qualified informants were interviewed, most of them sellers of both sexes and various ages who demonstrated know the properties of the products that sell, and that guide consumers on their forms of employment. In all cases, reference samples were obtained by the authors and deposited in the ethnobotanical collections of the LEBA. Each sample was identified with an alphanumeric code. All the Chinese plant products correspond to packaged dry materials, sometimes fragmented or powdered, corresponding to aerial parts: stems, leaves, barks, flowers, inflorescences, fruits, and seeds, and underground organs such as rhizomes, bulbs, and roots. The species were identified based on the external morphological characters of the samples. The products acquired in the dietéticas include herbal materials and also capsules containing extracts of the species that are listed in the official labels. Descriptive and distributional data of the species were obtained from Flora of China (Wu et al. 1995-2013). For the correct scientific names, The Plant List database (2013) was consulted.

Uses linked to Chinese tradition corresponding to plant products sold in the supermarkets were defined based on data coming from: 1) interviews, 2) the products labels, 3) information available on the Internet. Then, all data were checked with the general available literature (Adams and Lien 2013; Chang et al. 2001; Foster and Yue 1992; Hempen and Fischer 2009; Leung et al. 2003; Li and Wei 2002; Liu et al. 2015c; Tang and Eisenbrand 2013; Wu 2005; Yang 2013; Zhu 1998). Data from all these sources are mostly coincident. It is noteworthy that the Internet constitutes a relevant source of information for the urban scenario. First, because the web constitutes an enhancer agent of the spread of knowledge, as was indicated above. Thus, 
the Internet information orients the strategies of selection and consume for many urban residents interested in obtaining new medicinal plant products. Second, the Internet can act as point of direct sale for some consumers. Although not has been considered in this sense for this study, informal searches have allowed us to find the direct sale of fruits of Lycium barbarum L., locally known as "goji" (see below) through some web pages. The role of the Internet within the field of Urban Ethnobotany is evidently complex and requires deeper reflections.

\section{Review work}

This contribution is complemented by a review of the literature about biological activity and effects evaluated for each species considered. This kind of revision was also performed in previous works (Arenas et al. 2015; Hurrell et al. 2016, 2015a, 2015b; Puentes and Hurrell 2015), and it is pertinent because two main reasons: 1) It is useful information to know what uses have academic support, and/or what uses require more validation studies, 2) The uses assigned by people can proceed from the scientific knowledge diffused in the media, like Internet. This is a relevant aspect for plant products that access to the general commercial circuit. For the literature review various websites, especially the PubMed database (2016), were consulted.

\section{Species excluded}

Some plant species belonging to the TCP, like Ginkgo biloba L., Panax ginseng C.A. Mey., and Zingiber officinale Roscoe, have not been included in this contribution because they are widely disseminated through various products in the local general commercial circuit, i.e., are visible species. The medicinal products of these species include herbal materials but, mainly, dietary supplements (capsules, tablets). Curiously, these products are usually not marketed in the Barrio Chino, probably because its broad distribution in local urban area implies that can be acquired anywhere.

\section{RESULTS AND DISCUSSION}

The Table 1 summarizes the results obtained for the 32 species treated, including:

1) the scientific names, botanical family, distribution, Chinese name and Latin denomination of the products, reference samples (alphanumeric code between brackets)

2) uses linked to traditions (flavor, temperature, and meridians that refer to the TCP are indicated in italic)

3) biological activity and effects evaluated (bibliographical sources between parenthesis). Products belonging to the general commercial circuit are indicated with an asterisk. 
Table 1. Plant species and products of the Traditional Chinese Phytotherapy (TCP) commercialized in the Ciudad Autónoma de Buenos Aires, Argentina.

\begin{tabular}{|c|c|c|}
\hline $\begin{array}{l}\text { Species and products } \\
\text { [samples] }\end{array}$ & Uses linked to traditions & Biological activity and effects evaluated \\
\hline $\begin{array}{l}\text { Amomum tsaoko } \\
\text { Crevost \& Lemarié }\end{array}$ & \multirow{2}{*}{\multicolumn{2}{|c|}{ 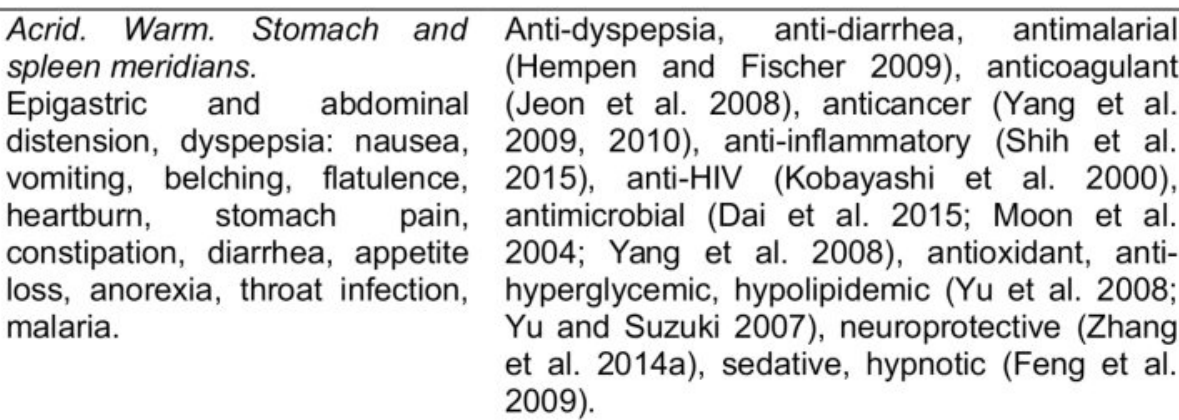 }} \\
\hline ao & & \\
\hline
\end{tabular}

Angelica sinensis (Oliv.) Diels

APIACEAE

China

\section{Dang gui}

Sinensis

Fragmented roots [H399]
Radix Angelicae
Sweet and acrid. Warm. Liver, hearth, and spleen meridians.

Blood deficiency: sallow complexion, pale nails, lips, and tongue, palpitations, dizziness, blood stasis: pain and numbness of extremities, anemia, hematoma, psoriasis, ulcerations, rheumatism, arthritis, inflammations, cramps, constipation, anxiety, stress, fatigue, premenstrual and menopausal symptoms, amenorrhea, menorrhagia dysmenorrhea, irregular menstruation, infertility, sexual female and male dysfunctions.
Hematopoietic, anti-anemia (Bradley et al. 1999), anti-atherosclerotic, anticoagulant, cardioprotective, vasorelaxant, antihypertensive, antioxidant, anti-inflammatory (Wu and Hsieh 2011; Zhang et al. 2010; Zhou et al. 2013), immuno-enhancer, antiviral, antimicrobial (Han and Guo 2012; Yang et al. 2012b), antisyphilitic (Drobnik 2016), anticancer (Gao et al. 2012a), hypocholesterolemic, hypolipidemic (Hurrell et al. 2015b), antiulcerative (Wong et al. 2008), neuroprotective: Alzheimer, dementia, mnemonic, antidepressant, sedative, antiinsomnia, anxiolytic (Hurrell et al. 2015a), adaptogenic: anti-fatigue (Liu et al. 2011), emmenagogue (Aramwit and Wirotsaengthong 2013; He et al. 1986), estrogenic (Circosta et al. 2006), sexual enhancer: female sexual dysfunction (Mazaro-Costa et al. 2010), premature ejaculation and erectile dysfunction (Hazard-Vallerand et al. 2016; Hsu et al. 2015).

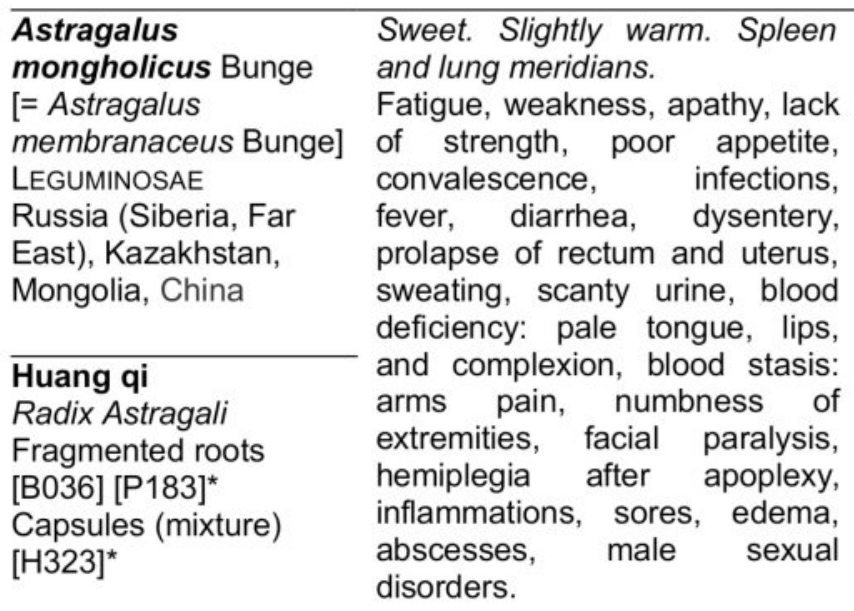

Adaptogenic: anti-fatigue (Liu et al. 2011), tonic (Asea et al. 2013), antioxidant, immunomulatory, antimicrobial, antiviral, anticancer, hematopoietic, hypotensive, antiinflammatory, hepatoprotective, antidiabetic (Jin et al. 2014; Li et al. 2004; McKenna et al. 2002), hypolipidemic, hypocholesterolemic, anti-atherosclerotic (Hurrell et al. 2015b), gastro and cardiovascular protective (Chen et al. 2016c; Ma et al. 2013), anti-osteoporosis (Jung Koo et al. 2014), emmenagogue (He et al. 1986), nephroprotective (Zhong et al. 2013), neuroprotective (Hurrell et al. 2015a), antidepressant, anticonvulsant, anxiolytic, memory and learning enhancer, male fertility enhancer (Singh et al. 2013b), sexual enhancer: erectile dysfunction (Hsu et al. 2015). 


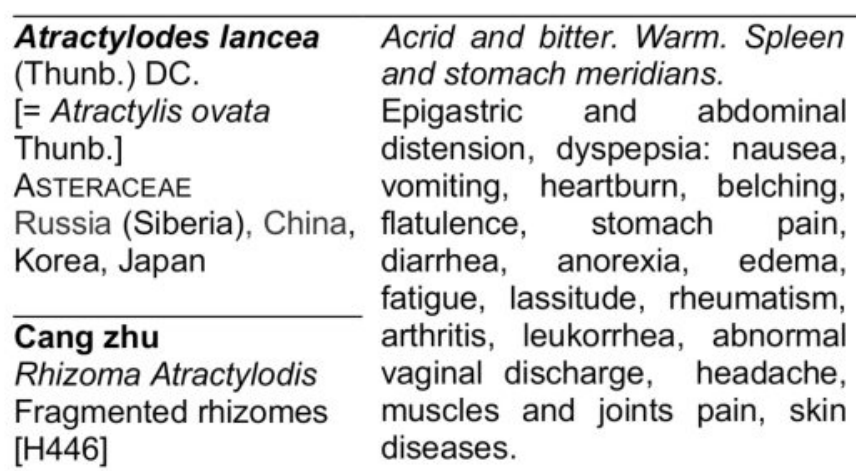

$\begin{aligned} & \text { Gastro-intestinal } \\ & \text { antiulcerative, } \\ & \text { anticancer, } \\ & \text { hypotensilive, } \\ & \text { antipyretic, }\end{aligned} \begin{array}{r}\text { stimulant, } \\ \text { antiplatelet, } \\ \text { antimicrobial }\end{array}$

(Koonrungsesomboon et al. 2014), anti-HIV

(Kato et al. 2012), hypolipidemic (Hurrell et al.

2015b), anti-obesity (Kang et al 2013b),

antioxidant, analgesic, anti-inflammatory

(Kakatum et al. 2012), hypoglycemic, antidiabetic ( $\mathrm{Li}$ et al. 2004; Zhu 1998), antieczema (Chan et al. 2008), anti-leucorrhea (Sakamoto et al. 1996), neuroprotective: Alzheimer, dementia, anti-epileptic, anxiolytic, anti-insomnia, memory and learning enhancer (Hurrell et al. 2015a).

\begin{tabular}{l} 
Chrysanthemum \\
morifolium Ramat. \\
[= Chrysanthemum \\
grandiflorum (Desf.) \\
Dum. Cours.] \\
AsTERACEAE \\
China \\
\hline Ju Hua \\
Flos Chrysanthemi \\
Morifolii \\
Inflorescences [P239] \\
[H420]*
\end{tabular}

Sweet and bitter. Mild cold. Lung and liver meridians.

Fever, headache, muscles spasms, dizziness, vertigo, cold, flu, cough, allergy, upper respiratory tract infections, pharyngolaryngitis, tonsillitis, hypertension, blood and kidney deficiency, blurred vision, conjunctive congestion with pain and swelling, liver hyperactivity, inflammations, tumors, swellings, furuncles, skin sores.

Antioxidant, anticancer, antimutagenic, cytotoxic, cardiovascular protective, antiarrhythmic, anti-atherosclerotic, antimicrobial, antiviral, anti-HIV, anti-allergic, antihepatotoxic, anti-inflammatory, antihypertensive, vasorelaxant, antipyretic, antiaging, neuroprotective: Parkinson, antiinsomnia (Hurrell and Puentes 2013; Lim 2013; Lin et al. 2015a; Lin and Harnly 2010; Miyazawa and Hisama 2003; PérezHernández et al. 2016; Sun et al. 2010; Zheng et al. 2015a, 2015b), antidiabetic (Yamamoto et al. 2015), hepatoprotective, hypocholesterolemic, anti-hyperlipidemic (Choo et al. 2004; Cui et al. 2014), ophthalmic (Terashima et al. 1991), antimelanogenic (Gui et al. 2014).

Cinnamomum cassia Acrid and sweet. Warm. Heart, (L.) J. Presl

LAURACEAE

China

\section{Rou gui}

Cortex Cinnamomi

Cassiae

Fragmented barks

[B001]

Powdered bark [H454] lung, and bladder meridians.

Kidney, liver, and spleen deficiency, fever, appetite loss, abdominal distension, dyspepsia: nausea, vomiting, flatulence, belching, heartburn, diarrhea, blood deficiency: pale complexion, faint pulse, edema, lack of sweating, abscesses, sores, extremities, knees, and lumbar pain, dribbling urine, profuse night urination, enuresis, infertility, sexual disorders: impotence, frigidity, nocturnal emission, spermatorrhea, dysmenorrhea, amenorrhea.
Anti-dyspepsia, antiulcerative, neuroprotective, hypotensive, vasorelaxant, antimicrobial (Zaidi et al. 2015; Zhu 1998), antiviral (Yeh et al. 2013), immunomulatory (Lee et al. 2011), cardiovascular protective (Song et al. 2013), antiplatelet (Kim et al. 2010), antioxidant (Yang et al. 2012a), anticancer (Chou et al. 2013; Kim et al. 2015a; Ngoc et al. 2014), osteoblastic (Lee and Choi 2006), antidiabetic (Jia et al. 2003; Yan et al. 2015), anti-obesity (Han et al. 2013), anti-inflammatory (Liao et al. 2012), antipyretic (Kurokawa et al. 1998), hypocholesterolemic, anti-dyslipidemia (Rahman et al. 2013), anti-dermatitis (Shin et al. 2015), anti-Alzheimer (Santos-Neto et al. 2006), antidepressant (Zada et al. 2016), anxiolytic (Jung et al. 2012), sexual enhancer: erectile dysfunction (Goswami et al. 2013, 2014), anti-dysmenorrhea, oligo/amenorrhea (Arentz et al. 2014; Marzouk et al. 2013). 


\begin{tabular}{ll}
\hline $\begin{array}{l}\text { Codonopsis pilosula } \\
\text { (Franch.) Nannf. }\end{array}$ & $\begin{array}{l}\text { Sweet. Neutral. Spleen and } \\
\text { lung meridians. }\end{array}$ \\
CAMPANULACEAE & $\begin{array}{l}\text { Stomach ailments, loose stool, } \\
\text { Russia (Far East), } \\
\text { Mongolia, China, Korea }\end{array}$ \\
& $\begin{array}{l}\text { diarrea, lack of appetite, } \\
\text { anorexia, cough, asthma, } \\
\text { wheezing, and dyspnea due to }\end{array}$ \\
\hline Dang shen & lung deficiency, blood \\
Radix Codonopsis & deficiency: palpitations, pale \\
Fragmented roots & complexion, lips, and tongue, \\
[P242] & dizziness, weakness of arms \\
& and legs, aging, male sexual \\
disorders, stress (tonic: similar & to Panax ginseng, but with \\
& shorter and milder effect).
\end{tabular}

Codonopsis pilosula Sweet. Neutral. Spleen and ranch.) Nannf.

Russia (Far East)

Mongolia, China, Korea

Radix Codonopsis

Fragmented roots
Tonic (Asea et al. 2013), adaptogenic: antistress, anti-aging, anti-anorexia, antipyretic, analgesic, hematopoietic, procoagulant, hypotensive, cardiovascular stimulant (Gao et al. 2012b), antioxidant, immunomodulatory, antimicrobial (Luo et al. 2007; $\mathrm{Ng}$ et al. 2004; Zheng et al. 2014b), hepatoprotective (Liu et al. 2015a), antiulcerative (Wang et al. 1997), chronic obstructive pulmonary disease (Shergis et al. 2015), antidiabetic ( $\mathrm{He}$ et al 2011), anticancer (Xin et al. 2012; Yang et al. 2013a), memory, concentration, and learning enhancer, neuroprotective (Hurrell et al. 2015a), menopause symptoms (Mayo 1999), menorrhagia (Tu et al. 2009), sexual enhancer: erectile dysfunction (Mahajan and Gajare 2012).

Coix lacryma-jobi L.
POACEAE
China, Bhutan, Nepal,
India, Sri Lanka,
Taiwan, Philippines,
South East Asia, New
Guinea

\section{Yi yi ren}

Semen Coicis

Lachryma-Jobi

Seeds [H307]
Sweet. Mild cold. Spleen, stomach, and lung meridians.

Spleen deficiency, diarrhea, dyspepsia: nausea, vomiting, indigestion, irritability, warts, chapped skin, abscesses, headache, neuralgia, lumbar and tendons pain, muscles spasms, knee weakness, pain and swelling in extremities, rheumatism, arthritis, gout, dysuria, syphilis, infections, abnormal vaginal discharges, fever, physical fatigue.
Adaptogenic: anti-fatigue, antimicrobial, antioxidant, immuno-enhancer, anticancer, antimutagenic, cytotoxic, abortifacient, antiprogestogenic, diuretic, hypolipidemic, antiobesity, anti-hyperglycemic, antidiabetic, antiallergic, prebiotic (Chen et al. 2011; Li 2006; Li et al. 2004; Lu et al. 2011; Manosroi et al. 2016), anti-inflammatory, antinociceptive (Choi et al. 2015; Sreekeesoon and Mahomoodally 2014), gastroprotective, anti-emetic, antinausea (Chen et al. 2016c), anti-adipogenesis (Kato et al. 2015b), anti-dysmenorrhea: analgesic (Zhang et al. 2000), nephroprotective, anti-hyperuricemia (Taejarernwiriyakul et al. 2015), antisyphilitic (Drobnik 2016)

\section{Dioscorea oppositifolia L.} [= Dioscorea opposita Thunb.]

DIOSCOREACEAE India, Bangladesh, Myanmar, Sri Lanka

\begin{tabular}{l}
\hline Shan yao \\
Rhizoma Dioscoreae \\
Oppositae \\
Fragmented rhizomes \\
[B031]
\end{tabular}

\section{Eleutherococcus} senticosus (Rupr. \& Maxim.) Maxim. [= Acanthopanax senticosus (Rupr. \& Maxim.) Harms] ARALIACEAE Russia (Siberia, Far East), China, Korea, Japan
Sweet. Neutral. Spleen, lung, and kidney meridians.

Lung deficiency, asthma, cough, dyspnea, spleen disorders, appetite loss, anorexia, thirst, sweating, fatigue, loose stool, chronic diarrhea, enuresis, frequent urination, polyuria, urinary incontinence, leukorrhea, spermatorrhea, and diabetes due to kidney disorders.
Antioxidant (Paulpriya and Mohan 2012), antiobesity (Jeong et al. 2016), antidiabetic (Fan et al. 2015; Jia et al. 2003), cardioprotective (Chen et al. 2015), anti-hypertensive (Nagai et al. 2014), anticancer (Chan and $\mathrm{Ng} \mathrm{2013),}$ hypocholesterolemic, immunomodulatory, antiinflammatory, estrogenic (Huang et al. 2010), menopausal syndrome (Wong et al. 2015), progestogenic, anti-progestogenic (Ahmed et al. 2014), female infertility (Ju et al. 2014), neuroprotective (Liu et al. 2013b), memory and learning enhancer (Hsieh et al. 2000), antidepressant (Yeung et al. 2014b).

Acrid and bitter. Warm. Liver
and kidney meridians.

General weakness, physical and/or mental weariness, lack of energy, vigour loss, poor resistance to diseases, adverse stress effects (Panax ginseng substitute), appetite loss, muscles and bones lacking strength, lower back, kidney,

Adaptogenic: anti-fatigue, antipyretic,
hypocholesterolemic, choleretic (Davydov and Krikorian 2000; Provino 2010), antioxidant, immunomodulatory, anti-inflammatory, antiulcerative, hypoglycemic, hepatoprotective, anti-osteoporosis, anti-allergic, anticancer, antiviral, antimicrobial (Huang et al. 2011; Tang and Eisenbrand 2013), antinephritic (Wojcikowski et al. 2009), vasorelaxant (Kwan 


\section{Ci wu jia}

Radix Eleutherococci

Fragmented roots and rhizomes [RF05]

[P186] ${ }^{*}$

Capsules (mixture)

[P170 $]^{*}$ loins, and knees pains, edema, rheumatism, arthritis, cough, bronchitis, lung ailments, tumors, insomnia, anxiety, concentration and memory loss, sexual disorders.

et al. 2004), cardioprotective (Maslov et al. 2009), anti-edema (Fukada et al. 2016), antitussive (Barth et al. 2015), neuroprotective: Parkinson, ischemia, learning and memory enhancer, antidepressant, anxiolytic (Hurrell et al. 2015a), male anti-infertility (Nantia et al. 2009), sexual desire (libido) enhancer (Debnath et al. 2011), erectile dysfunction due to depressive conditions (Rajput et al. 2011)

\begin{tabular}{ll}
\hline Eucommia ulmoides & $\begin{array}{l}\text { Sweet. Warm. Liver and kidney } \\
\text { Oliv. }\end{array}$ \\
meridians. \\
EuCOMmIACEAE & Aging, hypertension, vertigo, \\
China & back, waist, ankles, and knees \\
\hline Du zhong & pain, muscles, tendons, bones, \\
Cortex Eucommiae & and ligaments weakness, \\
Fragmented barks & arthritis, swelling, frequent \\
[H447] & urination, overweight, wetness \\
& and itching of genitals, male \\
& sexual disorders: premature \\
& ejaculation, impotence, fetal \\
& movement, placental leakage, \\
& stress, fatigue.
\end{tabular}

Anti-aging, antioxidant, anti-obesity, antidiabetic, immunomodulatory, antimicrobial, anti-allergic, hypotensive, anti-inflammatory, anti-osteoporosis, anticancer, cardioprotective, sexual enhancer: erectile dysfunction ( $\mathrm{He}$ et al. 2014; Hou and Jin 2005; Hussain et al. 2016), hepatoprotective (Lee et al. 2014), hypolipidemic, hypocholesterolemic, antiatherosclerotic (Hurrell et al. 2015b), progestogenic, anti-progestogenic (Ahmed et al. 2014), osteoblastic (Lee et al. 2016b), antiosteoarthritis (Xie et al. 2015), memory and learning enhancer, neuroprotective: Parkinson, Alzheimer, antidepressant (Hurrell et al. 2015a), nephroprotective ( $\mathrm{Li}$ et al. 2012), adaptogenic: anti-fatigue (Panossian 2003).

\section{Fallopia multiflora} (Thunb.) Haraldson [= Polygonum multiflorum Thunb., Reynoutria multiflora (Thunb.) Moldenke]

POLYGONACEAE

China, Japan, Taiwan

\section{He shou wu}

Radix Polygoni

Multiflori

Fragmented roots [B016]
Slightly bitter and sweet. Warm. Liver and kidney meridians.Aging, hair graying, alopecia, vertigo, insomnia, dizziness, tinnitus, neurasthenia, skin eruptions, itching, skin sores, furuncles, constipation, blood and bowels deficiency, fever, anemia, lower back and knees pain and weakness, lymphatic tuberculosis, inflammations, scrofula, tumors, malaria, leukorrhea, night sweating, spermatorrhea, prostatitis, weak seminal emission, male infertility, male sexual disorders: impotence.
Tonic (Asea et al. 2013), anti-aging hematopoietic, antihyperlipidemic, antidyslipidemia, hepatoprotective, antiatherosclerotic, cardiovascular protective, nephroprotective, immunomodulatory, antibacterial, intestinal peristalsis improver, anti-bronchitis, anti-inflammatory, memory and learning enhancer, neuroprotective: neurodegenerative disorders, antipyretic (Foster and Yue 1992; Hou and Jin 2005; Lin et al. 2015b; Ling and Xu 2016; Sun et al. 2016), antidiabetic (Jia et al. 2003), antioxidant (Lv et al. 2014), anticancer (Zhu et al. 2016a), anti-HIV (Lin et al. 2010), hair growth (antialopecic), hair blackening (Han et al. 2015; Park et al. 2011), antidepressant (Wang and Zhu 2012), sedative, anti-insomnia, anxiolytic (Bounda and Feng 2015), spermatogenesis enhancer (Chen et al. 2016a), sexual enhancer: erectile dysfunction (Lee et al. 2015c; Singh et al. 2010). 
Fritillaria cirrhosa D.

Don

LILIACEAE

China, Nepal, Bhutan, India

\section{Chuan bei mu}

Bulbus Fritillariae

Cirrhosae

Bulbs [H455]
Bitter. Slightly cold. Lung and heart meridians.

Chronic cough, asthma, dry cough and hemoptysis, dry throat, scanty and bloody phlegm, difficult expectoration pyemesis, cold, bronchitis, pneumonia, chest and heart tightness, atelectasis, lung abscess, pharyngolaryngitis, pulmonary tuberculosis, swelling, scrofula, goiter, mastitis, furuncles, ulcerations, fever, hypertension, low appetite, dysphoria.
Antitussive, anti-asthmatic, expectorant, phlegm-relieving, tracheobronchial relaxation, upper respiratory tract infections, antituberculosis, anti-inflammatory, antispasmodic, analgesic, diuretic, anti-hypertensive, antipyretic (Chan et al. 2000, 2011; Chang et al. 2001; Khare 2008; Schröder et al. 2013; Wang et al. 2011, 2016a, 2016b; Wardlaw et al. 1988; Yeum et al. 2007), antioxidant (Li et al. 2013b), anti-HIV (Lam et al. 2000), anticancer (Bokhari and Syed 2015; Kavandi et al. 2015; Wang et al. 2014a).

\section{Gastrodia elata Blume ORCHIDACEAE Russia (Siberia, Far East), China, Korea, Japan, Nepal, Bhutan, India, Taiwan}

Tian ma
Rhizoma Gastrodiae
Elatae
Fragmented rhizomes
[H449]

Sweet. Neutral. Liver meridian.

Liver disorders, dizziness, vertigo, seizures in children, epilepsy, tetanus, paralysis, hemiplegia, neurasthenia, blood stasis: numbness and pain in the extremities, rheumatism, arthritis, muscles spasms, movement restriction, neuralgia, headache, neurodegenerative disorders, male sexual disorders: impotence, aging, lassitude, fatigue.
Antioxidant, anti-aging, analgesic, anti-vertigo, anti-epileptic, anticonvulsive, antidepressant, sedative, hypnotic: anti-insomnia, anxiolytic, memory and learning enhancer, antipsychotic, neuroprotective, neurodegenerative disorders: Alzheimer, Parkinson, antiviral, antiinflammatory, cardiovascular protective, anticancer (Hou and Jin 2005; Hurrell et al. 2015a; Zhan et al. 2016), gastroprotective (An et al. 2007), bone protector, anti-osteoporosis (Huang et al. 2015b; Zheng et al. 2014a), antimigraine (Wang et al. 2015b), antidiabetic (Yang et al. 2016a), anti-asthmatic (Jang et al. 2010), sexual enhancer: erectile dysfunction (Lee et al. 2015c), adaptogenic: anti-fatigue (Zhaobao and Bing 2010).

Antitussive, expectorant, antipyretic (Hou and Jin 2005), antioxidant, anticoagulant, blood circulation enhancer, vasorelaxant, immunomodulatory, antidiabetic, anti-epileptic, anticonvulsive, anti-amnesia (Gairola et al. 2010; Luszczki et al. 2009; $\mathrm{Ng}$ et al. 2004; Su et al. 2013), anti-HIV, anticancer, antiproliferative (Kong et al. 2010; de la Cruz et al. 2015; Um et al. 2010), antibacterial, antifungal (Matsuura et al. 1996), antiinflammatory (Yoon et al. 2010a, 2010b), analgesic (Ishikawa et al. 2001).

\begin{tabular}{ll}
\hline Glehnia littoralis F. & $\begin{array}{l}\text { Sweet. Slightly cold. Lung and } \\
\text { stomach meridians. }\end{array}$ \\
Schmidt ex Miq. & $\begin{array}{l}\text { Chronic bronchitis, pulmonary } \\
\text { APIACEAE }\end{array}$ \\
Russia (Siberia, Far & $\begin{array}{l}\text { tuberculosis, dry cough or } \\
\text { East), China, Korea, } \\
\text { cough with scanty sputum, } \\
\text { Japan, Taiwan }\end{array}$ \\
\hline hemoptysis, hoarseness, dry \\
throat, dry mouse, fatigue, chest \\
Radix Glehniae & pain, rheumatism, arthritis, \\
Fragmented roots & joints and muscles pain, \\
[B041] & migraine, dry tongue, poor \\
& appetite, constipation, body \\
fluids disorders, thirst, sweating, \\
febrile diseases.
\end{tabular}

Glycyrrhiza uralensis

Fisch.

LEGUMINOSAE

Russia (Siberia),

Kazakhstan, Mongolia,

China, Kyrgyzstan,

Tajikistan, Afghanistan, Pakistan
Sweet. Neutral. Heart, spleen, lung, and stomach meridians. Respiratory disorders, cough, asthma, phlegm, dyspnea, bronchitis, sore throat, painful swellings, seizures, spleen and stomach dysfunctions, gastrointestinal spams, abdominal
Antitussive, anti-asthmatic, antioxidant, antiinflammatory, antirheumatic, antithrombotic, hepatoprotective, anti-atherosclerotic, antiallergic, antimicrobial, antiviral, antimalarial, sweetener, antacid, antiulcerative, antispasmodic, anticancer (Foster and Yue 1992; Ji et al. 2016; Kim et al. 2012; Tang and 


\begin{tabular}{ll}
\hline Gan cao & painful stiffness, stomach and \\
Radix Glycyrrhizae & duodenal ulcerations, hepatitis, \\
Uralensis & muscle spasms, infections, \\
Fragmented roots & inflammations, skin diseases, \\
[B040] & furuncles, abscesses, sexual \\
& disorders, hysteria, arrhythmia, \\
& palpitations, general weakness, \\
& poor appetite, lassitude, stress, \\
& fatigue.
\end{tabular}

$\begin{array}{ll}\text { Houttuynia cordata } & \begin{array}{l}\text { Acrid. Slightly cold. Lung } \\ \text { meridian. }\end{array}\end{array}$

SAURURACEAE

China, Korea, Japan,

Taiwan, Bhutan, Nepal, India, Myanmar,

Thailand, Indonesia

Respiratory disorders, chest pain, lung abscess with purulent expectoration, cough, dyspnea, rhinitis, sinusitis, pneumonia, bronchitis, inflammations, skin diseases: psoriasis, furuncles, skin sores, measles, epilepsy, nephritis, dysuria, urinary infections, leukorrhea, fever with chills, rheumatism, dysentery,

\section{Yu xing cao}

Herba Houttuyniae

Fragmented aerial parts [B006] hemorrhoids, edema, stress, fatigue, male sexual disorders: impotence.
Eisenbrand 2013), cardioprotective (Liu et al. 2014), progestogenic, anti-progestogenic (Ahmed et al. 2014), antisyphilitic (Drobnik 2016), detoxificant (Peter et al. 2013), immunomodulatory (Ayeka et al. 2016), antidermatitis (Cha et al. 2016), anti-emetic, gastroprotective (Chen et al. 2016c), neuroprotective: Alzheimer, Parkinson (Link et al. 2015; Zarmouh et al. 2016), antidepressant (Rajput et al. 2011), sexual desire (libido) enhancer (Yarnell and Abascal 2013), adaptogenic: anti-fatigue (Corneanu and Corneanu 2013; Shang et al. 2010).

Antitussive, anti-bronchitis, anti-sinusitis, severe acute respiratory syndrome, immunomodulatory, antimicrobial, antiviral, anti-allergic, diuretic, vasorelaxant, antihypertensive, anti-dermatitis, antiinflammatory, antipyretic, detoxificant, hepatoprotective, anti-obesity, anticancer (Fu et al. 2013; Kumar et al. 2014a; Tang and Eisenbrand 2013; Zhu 1998), antioxidant (Doi et al. 2014), adaptogenic: physical endurance (Yang et al. 2015b), anthelmintic (Vijaya and Yadav 2016), anti-hyperglycemic (Kumar et al. 2014b), nephroprotective (Kang et al. 2013a), anti-epileptic, antidepressant, neuroprotective (Huh et al. 2014; Jeon et al. 2014; Kim et al. 2015b), sexual enhancer: erectile dysfunction linked to prostatic hyperplasia, anti-prostatitis, androgenic (Kumar et al. 2011; Yarnell and Abascal 2005).

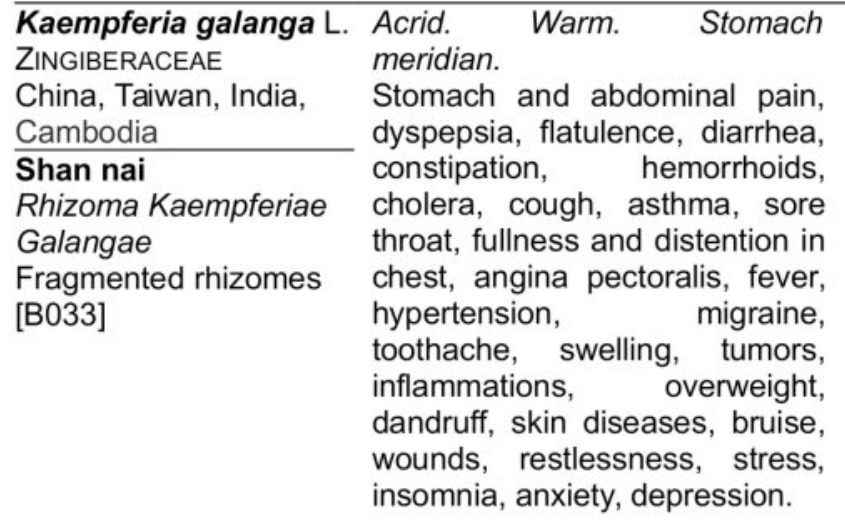

Antitussive (Gairola et al. 2010), antihypertensive, vasorelaxant, cardiotonic, anticoagulant, diuretic, anti-inflammatory, analgesic, antinociceptive, antioxidant, anthelmintic, digestive, antipyretic, antiallergic, antidiabetic, antimicrobial, antituberculosis, antiviral, expectorant, anticancer, cytotoxic, central nervous system stimulant, anti-dermatitis, cicatrizant, insect repellent, larvicidal (Kumar 2014; Lim 2016; Preetha et al. 2013; Singh et al. 2013a; Umar et al. 2011), osteoblastic (Guo et al. 2012); antimalarial (Thiengsusuk et al. 2013), anti-obesity (Lee et al. 2015b), hypolipidemic, hypocholesterolemic, anti-atherosclerotic (Achuthan and Padikkala 1997), anxiolytic, sedative, anti-insomnia (Edewor-Kuponiyi 2013). 


\begin{tabular}{|c|c|}
\hline $\begin{array}{l}\text { Leonurus japonicus } \\
\text { Houtt. } \\
\text { LAMIACEAE } \\
\text { China, Korea, Japan, } \\
\text { Taiwan, South East Asia } \\
\text { Yi mu cao } \\
\text { Herba Leonuri Japonici } \\
\text { Fragmented aerial parts } \\
\text { [B020] }\end{array}$ & $\begin{array}{l}\text { Acrid and bitter. Slightly Cold. } \\
\text { Heart, liver, and bladder } \\
\text { meridians. } \\
\text { Blood stasis: pain and } \\
\text { numbness of extremities, } \\
\text { hypertension, coronary } \\
\text { diseases, hematuria, dysuria, } \\
\text { oliguresis, dysmenorrhea, } \\
\text { amenorrhea, menorrhagia, } \\
\text { abdominal and pelvic pain, } \\
\text { difficult labor, edema, skin } \\
\text { sores, anxiety, depression, } \\
\text { tiredness, insomnia. }\end{array}$ \\
\hline
\end{tabular}

\begin{tabular}{l}
\hline Ligusticum striatum \\
DC. [= Ligusticum \\
chuanxiong S.H.Qiu, \\
Y.Q.Zeng, K.Y.Pan, \\
Y.C.Tang \& J.M.Xu; \\
= Ligusticum wallichii \\
Franch.] \\
APIACEAE \\
China, Kashmir, Nepal, \\
India
\end{tabular}

Chuan xiong

Rhizoma Ligustici

Chuanxiong

Fragmented rhizomes

[B027]
Acrid. Warm. Gallbladder, liver, and pericardium meridians.

Cardiovascular disorders,

hypertension, blood stasis: pain and numbness of extremities, traumatic injury, irregular menstruation, dysmenorrhea, amenorrhea, abdominal pain, numbness muscles, tendons, and joints, arthritis, rheumatic arthralgia, vertigo, migraine, abscesses, syphilis, sexual dysfunction. angina pectoris, swelling due to
Cardioprotective, blood circulation enhancer, hypotensive, vasorelaxant, antiplatelet, antioxidant, antimicrobial, anticancer, diuretic, nephroprotective, anti-menorrhagia, uterotonic, emmenagogue, neuroprotective, metabolic syndrome (Foster and Yue 1992; Kuchta et al. 2012; Shang et al. 2014; Yang et al. 2014), hepatoprotective, cytoprotective, cytotoxic (Jiang et al. 2015; Moon 2010), analgesic, antiinflammatory (Khan et al. 2012), anxiolytic, antidepressant (Rauwald et al. 2015), female fertility enhancer (Hung et al. 2016).

Cardioprotective, antithrombotic, hypotensive, vasoprotective, anticancer, anti-atherosclerotic, hypocholesterolemic, muscle contractility enhancer, neuroprotective: neurodegenerative disorders: Alzheimer, anti-inflammatory, analgesic, immunomodulatory (Fu et al. 2015; Hu et al. 2015; Li et al. 2013a; Ran et al. 2011; Zhu 1998), antioxidant (Liu et al. 2015b), gastroprotective (Li et al. 2005), anti-migraine (Wang et al. 2015b), anti-asthmatic (Wei et al. 2016), nephroprotective (Yang et al. 2011), anti-hyperglycemic, antidiabetic (Li et al. 2004; Shih et al. 2015), progestogenic (Lim et al. 2006), anti-dysmenorrhea (Hsu et al. 2006), antisyphilitic (Drobnik 2016), sexual enhancer: erectile dysfunction (Xiao et al. 2010).

\begin{tabular}{l} 
Lilium brownii F.E.Br. \\
ex Miellez \\
LILIACEAE \\
China \\
\hline
\end{tabular}

Bai he

Bulbus Lilii Brownii

Bulb scales [B028]

Sweet. Slightly Cold. Heart and lung meridians.

Chronic cough, dry cough due to lung deficiency, pertussis, asthma, hemoptysis, sore throat, bronchitis, pulmonary tuberculosis, febrile diseases, dizziness, restlessness, rapid pulse, palpitations, and urinary disorders due to heart deficiency, fatigue, dreamdisturbed sleep, insomnia.

Antitussive, antioxidant, antidiabetic, anticancer, cytotoxic, immuno-enhancer, antiinflammatory, antiplatelet, sedative, antiinsomnia (Mimaki et al. 1995, 2001; Sun et al. 2014; Wang et al. 2015c; Wong et al. 2006), respiratory system diseases (Han et al. 2008), antimicrobial (Yeo and Tham 2012), antifatigue (He et al. 2009), anti-aging, antiamnesia, neuroprotective: neurodegenerative disorders, anticonvulsive (Lin et al. 2003), antidepressant (Zhang et al. 2014b).

Sweet. Cold. Lung, stomach, and large intestine meridians.

Thunb.

CAPRIFOLIACEAE

China, Korea, Japan,

Taiwan

Anti-asthmatic (Hong et al. 2013), upper respiratory tract infection (Lu et al. 2016), antiseptic, anti-bronchitis (Kim et al. 2015c; Ko et al. 2013), anti-allergic, anti-dermatitis (Tian et al. 2012), antiviral, antimicrobial, antioxidant, cardioprotective, analgesic, anti-inflammatory, antipyretic, hypolipidemic, hepatoprotective, cytoprotective (Mahboob et al. 2016; Ryu et al. 2010; Shang et al. 2011), anticancer (Han et al. 2016), anti-hypertensive (Cheng et al. 1944), antidiabetic, anti-hyperglycemic, antiobesity (Lee et al. 2016a), antinephritic (Tzeng et al. 2014), anti-arthritis (Huh et al. 2012), 


\begin{tabular}{|c|c|}
\hline $\begin{array}{l}\text { Jin yin hua } \\
\text { Flos Lonicerae } \\
\text { Japonicae } \\
\text { Buds and flowers } \\
\text { [B017] }\end{array}$ & \\
\hline $\begin{array}{l}\text { Lycium barbarum } \mathrm{L} \text {. } \\
\text { SOLANACEAE }\end{array}$ & $\begin{array}{l}\text { Sweet. Neutral. Liver, kidney, } \\
\text { and lung meridians. }\end{array}$ \\
\hline China & $\begin{array}{l}\text { General weakness, lack of } \\
\text { energy, fatigue, lassitude, }\end{array}$ \\
\hline $\begin{array}{l}\text { Gou qi zi } \\
\text { Fructus Lycii } \\
\text { Fruits [H037] [D001] } \\
\text { [R169]* }^{*}\end{array}$ & $\begin{array}{l}\text { stress, aging, poor vision, } \\
\text { cataract, diminished visual } \\
\text { acuity, blood disorders, anemia, } \\
\text { diabetes, back and joints pains, } \\
\text { lung deficiency, chronic cough, } \\
\text { tinnitus, headache, neuralgia, } \\
\text { dizziness, burns, ulcerations, } \\
\text { skin sores, furuncles, nocturnal } \\
\text { emission, sperm production } \\
\text { deficiency, male infertility, male } \\
\text { sexual disorders: impotence. }\end{array}$ \\
\hline
\end{tabular}

Flos Lonicera

Japonicae

Buds and flowers

Lycium barbarum $\mathrm{L}$.

SOLANACEAE

Gou qi zi

Fructus Lyci

Fruits [H037] [D001]

cognitive protective, neuroprotective (Weon et al. 2011; Yu et al. 2015), gastroprotective (Huh et al. 2011), bowel diseases (Park et al. 2013), antisyphilitic (Drobnik 2016).

Adaptogenic: anti-stress, antioxidant, antiaging, memory and learning enhancer, neuroprotective, anxiolytic, antidepressant, cardioprotective (Hurrell et al. 2013, 2015a), anti-osteoporosis (Jin et al. 2013), anti-arthritis (Liu et al. 2015d), hepatoprotective (Xiao et al. 2012), hypolipidemic, hypocholesterolemic, anti-atherosclerotic (Hurrell et al. 2015b), antiobesity (Amagase and Nance 2011), nephroprotective, antidiabetic (Du et al. 2016; Li et al. 2004), immunomodulatory (Amagase et al. 2009), anti-inflammatory (Nardi et al. 2016), anticancer (Mao et al. 2011), spermatogenesis enhancer (Qian and Yu 2016), sexual enhancer: erectile dysfunction, testicular and prostatic protective (Patel et al. 2011; Semwal et al. 2013; Sohn et al. 2008).

Nelumbo nucifera
Gaertn.
NELUMBONACEAE
Russia (Siberia, Far
East), China, Korea,
Japan, Nepal, Bhutan,
Pakistan, India, Sri
Lanka, Philippines,
South West Asia, South
East Asia, New Guinea,
Australia

Lian zi

Semen Nelumbinis

Seeds [BH20]

Lian zi xin

Plumula Nelumbinis

Plumules [B018]

\section{Sweet. Neutral. Spleen, kidney} and heart meridians.

Enteritis, dyspepsia: nausea, vomiting, flatulence, diarrhea, poor appetite, anorexia, urinary incontinence, leukorrhea, abnormal vaginal discharge, menorrhagia, hypertension, arrhythmia, chest pain, sweating, dizziness, fever, respiratory infections, anxiety, insomnia, poor memory, amnesia, spermatorrhea, nocturnal emissions, male sexual disorders: premature ejaculation.
Antioxidant, antifertility, antipyretic hypotensive, anti-arrhythmic, antiviral, immunomodulatory, anti-asthmatic, antiarthritic, anti-inflammatory, anti-dermatitis, antiatherosclerotic, hepatoprotective, gastroprotective, anticancer (Paudel and Panth 2015), anti-diarrhea (Knipping et al. 2012), antithrombotic (Zhou et al. 2013), antibacterial (Tho et al. 2013), hypoglycemic (Kato et al. 2015a), hypolipidemic, hypocholesterolemic, anti-obesity (Liao and Lin 2013; You et al. 2014), bronchodilator (Tan et al. 2015), neuroprotective, memory and learning enhancer (Jung et al. 2015; Kim et al. 2014), anxiolytic, sedative, antidepressant (Sugimoto et al. 2010), anti-insomnia (Sirisha-Chowdary 2013), anti-estrogenic, antispermatogenic (Chauhan et al. 2009; Mutreja et al. 2008), sexual enhancer: erectile dysfunction (Chen et al. 2008).

Antioxidant, antitussive, cardiovascular
protective, anti-inflammatory, anticancer, immunomodulatory, antimicrobial ( Chen et al. 2016b; Hou and Jin 2005), antinephritic, antidiabetic, anti-hyperglycemic, antihyperlipidemic (Jia et al. 2003; Wang et al. 2015d), anti-obesity (Wang et al. 2014c), hepatoprotective (Lu et al. 2014), hematopoietic (Fleischer et al. 2016), antiosteoporosis (Huang et al. 2015a), antithrombotic (Kou et al. 2006), anti-aging (Sun et al. 2013), neuroprotective (Lin et al. 2015c), anxiolytic (Liu et al. 2015c), anti- 


\begin{tabular}{ll}
\hline Mai dong & insomnia (Zhao et al. 2008a), anti-fatigue \\
Radix Ophiopogonis & (Chen et al. 2010). \\
Japonici & \\
Fragmented roots & \\
[B039] &
\end{tabular}

Paeonia lactiflora Pall. Bitter and sour. Slightly cold. [= Paeonia albiflora Liver and spleen meridians.

Pall.]

PAEONIACEAE

Russia (Siberia, Far East), Mongolia, China, Korea, Japan

\section{Bai shao}

Radix Paeoniae

Lactiflorae

Fragmented roots [B036]
Liver hyperactivity and pain, gastric ulcers, night sweating, spermatorrhea, blood stasis: pain and numbness of extremities, spasms, abnormal vaginal discharge, irregular menstruation, amenorrhea, menorrhagia, mastitis, hypochondrium and abdomen pain, headache, diarrhea, dysentery, weakness, tinnitus, convulsions, dizziness, male sexual disorders: impotence.
Antioxidant, anti-inflammatory, antiviral, antibacterial, immunomodulatory, antidiabetic, cardiovascular protective, anti-atherosclerotic, anti-hyperlipidemic, anti-obesity ( $\mathrm{He}$ and Dai 2011; Li et al. 2004; Parker et al. 2016; Zhang et al. 2015), anticancer (Lam et al. 2015), antithrombotic (Ye et al. 2016), hematopoietic (Zhu et al. 2016b), anti-dermatitis (Sun et al. 2015), neuroprotective: Alzheimer (Fu et al. 2015), antinociceptive (Zhang et al. 2016), antidepressant (Farahani et al. 2015), uterine relaxant (Hehir and Morrison 2016), antiamenorrhea (Arentz et al. 2014), breast hyperplasia (Peng et al. 2015), sexual enhancer: erectile dysfunction, prostatic hyperplasia, androgenic (Hetchman 2013; Washida et al. 2009).

Prunella vulgaris L. Acrid and bitter. Cold.

\section{LAMIACEAE Liver meridian.}

Russia (Siberia), China, Liver deficiency, bloodshot and Korea, Japan, Taiwan, swollen eyes, painfull eyeballs, Kyrgyzstan, Uzbekistan, headache, vertigo, dizziness, Kazakhstan, Tajikistan, anxiety, phlegm, hypertension, Turkmenistan, Pakistan, hemostasis, inflammations, India, Bhutan, Nepal, lymphatic tuberculosis, South West Asia, Europe, Africa, North America

\section{Xia ku cao}

Spica Prunellae

Vulgaris

Fragmented

inflorescences [B025]
Antioxidant, anti-hypertensive, diuretic (Yang et al. 2013b), blood circulation enhancer, cardioprotective, phlegm-relieving, immunomodulatory, antiviral: anti-HIV, antiherpes, antimicrobial, anti-allergic, antiinflammatory, analgesic, anti-hyperglycemic, antidiabetic, antilipidemic, hypocholesterolemic, anti-atherosclerotic, antitussive, anticancer, hepatoprotective, systemic anaphylaxis inhibition, UVphotoprotective (Adams and Lien 2013; Hwang et al. 2012; Lam et al. 2000; Li et al. 2015; Meng et al. 2014), breast hyperplasia (Peng et al. 2015), anti-estrogenic (Collins et al. 2009), anti-arthritis (Jung et al. 2001), anticolitis (Haarberg et al. 2015), anxiolytic (Kwon et al. 2014), memory and learning enhancer, neuroprotective: Alzheimer, schizophrenia (Park et al. 2010, 2015). 


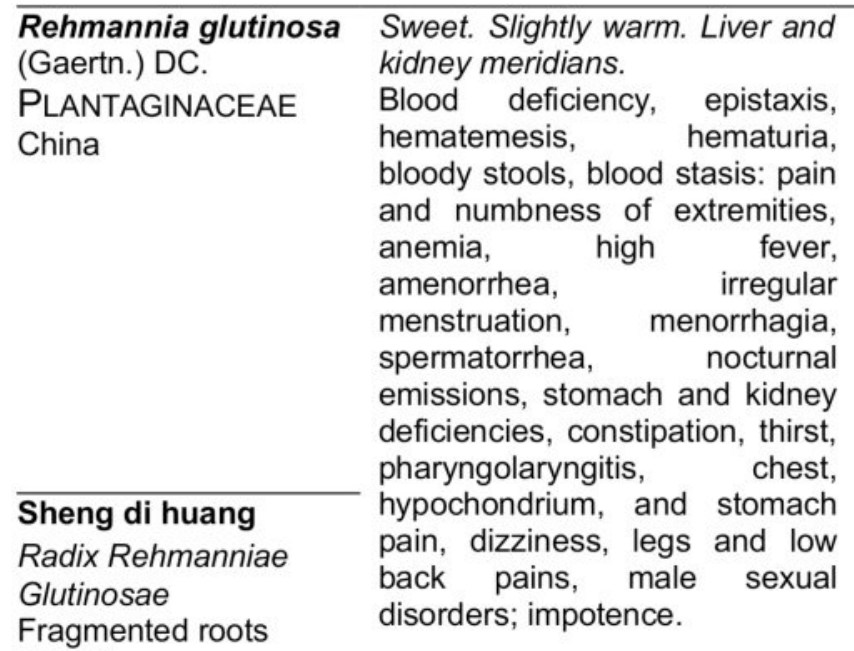
[H448]
Cardiovascular protective, hemostatic, antianemia, hematopoietic, diuretic, antiinflammatory (Chang et al. 2001; Fleischer et al. 2016), antidiabetic, hypoglycemic (Jia et al. 2003; Li et al. 2004; Zhang et al. 2004), nephroprotective (Hsu et al. 2014), hypocholesterolemic, hypolipidemic (Hurrell et al. 2015b), hepatoprotective (Liu et al. 2012), lung protective (Fu et al. 2014), anticancer (Jin et al. 2015), immune enhancer (Huang et al. 2013), anti-osteoporosis (Lim and Kim 2013), anti-dermatitis (Sung et al. 2011), analgesic (Wang et al. 2014b), antioxidant, adaptogenic, neuroprotective: Alzheimer, Parkinson, cerebral ischemia, memory and learning enhancer, antidepressant, anti-insomnia (Hurrell et al. 2015a), sexual enhancer: erectile dysfunction (Feng et al. 2012), prostatic hyperplasia (Shin et al. 2012).

\section{Scaphium affine}

(Mast.) Pierre

[= Sterculia

lychnophora Hance]

MALVACEAE

South East Asia

Pang da hai

Semen Sterculiae

Lychnophorae

Seeds [B035]
Sweet. Cold. Lung and large intestine meridians.

Chest pain, hoarseness, aphony, pharyngolaryngitis, tonsillitis, cough with viscous sputum, flu, vomiting, fever, mucous inflammation, skin eruption, headache, toothache, constipation, abdominal spasms, cramps, bones pain, bloodshot eyes, nose bleeds, hemorrhoids.
Anti-inflammatory, analgesic, neuroprotective: Alzheimer, laxative (Shetty et al. 2014; Wang et al. 2013; Wu et al. 2007), antifungal, antibacterial, anti-caries (Palve et al. 2015; Yang et al. 2016b), anti-obesity (Zhao et al. 2008b), immunomodulatory, intestinal peristalsis stimulant, antioxidant ( $\mathrm{Ai}$ et al. 2012), uterotonic, anti-menorrhagia, antiestrogenic, anticancer (Sharma et al. 2012).

\section{Schisandra chinensis}

(Turcz.) Baill.

SCHISANDRACEAE

Russia (Siberia, Far

East), China, Korea, Japan
Sour. Warm. Lung, kidney, and heart meridians.

Asthma, chronic cough due to lung deficiency, pulmonary asthenia, dyspnea, phlegm, weak pulse, spontaneous and night sweating, headache, liver disorders, hepatitis, chronic diarrhea due to spleen deficiency, dysentery, vomiting due to kidney deficiency, polydipsia, urinary incontinence, frequent urination, palpitations, restlessness, neurasthenia: lassitude, irritability, mental and physical fatigue, anxiety,
Anti-asthmatic, antitussive, respiratory stimulant, expectorant, antibacterial, antiallergic, eyesight enhancer, uterotonic, antidiarrhea, antidysenteric, cardiotonic, cardiovascular protective (Chang et al. 2001; Chen et al. 2013; Chun et al. 2014; Lee et al. 2015a), antiseptic (Kook et al. 2015), anticancer (Poornima et al. 2016; Qu et al. 2014), neuroprotective: neurodegenerative disorders, memory and learning enhancer, anxiolytic, anti-insomnia, antidepressant, sedative (Hurrell et al. 2015a), immunomodulatory, anti-inflammatory hepatoprotective, antidiabetic, antioxidant, detoxificant (Adams and Lien 2013; Li et al. 2004), hypocholesterolemic (Hurrell et al. 


\begin{tabular}{|c|c|}
\hline $\begin{array}{l}\text { Wu wei zi } \\
\text { Fructus Schisandrae } \\
\text { Fruits [RF59] [P208]* } \\
\text { Capsules (mixture) } \\
\text { [H323] }^{*}\end{array}$ & $\begin{array}{l}\text { nervousness, insomnia } \\
\text { spermatorrhea, male sexua } \\
\text { disorders: } \\
\text { premature ejaculation. }\end{array}$ \\
\hline
\end{tabular}

2015b), antiulcerative, anti-dermatitis (Foster and Yue 1992), anti-HIV (Xu et al. 2015), antifungal (Yang et al. 2015a), gastro-intestinal disorders (Ahn et al. 2015), adaptogenic: antifatigue (Asea et al. 2013; Mahajan and Gajare 2012; Panossian 2013, 2003), sexual enhancer: erectile dysfunction (Choo et al. 2014; Han et al. 2012).

Siraitia grosvenorii
(Swingle) C. Jeffrey ex
A.M. Lu \& Zhi Y. Zhang
[= Momordica
grosvenorii Swingle]
CUCURBITACEAE
China

Luo han guo

Fructus Momordicae Grosvenorii

Fruits [P245]

\author{
Sweet. Slightly cold. Lung and \\ spleen meridians. \\ Lung deficiency, bronchitis, \\ pulmonary tuberculosis, \\ phthisis, cough, asthma, \\ pharyngolaryngitis, phlegm, \\ sore trhroat, acuted tonsillitis, \\ aphony, dyspnea, cold, skin \\ deseases, psoriasis, furuncles, \\ gastritis, constipation, \\ overweight, diabetes, aging, \\ fatigue.
}

Antitussive, phlegm-relieving, antioxidant, hepatoprotective, immunomodulatory, antimicrobial, antiviral, antihistaminic, antiallergic, antidiabetic, sweetener (Jin and Lee 2012; Li et al. 2014), adaptogenic: anti-fatigue (Liu et al. 2013a), gastro-intestinal stimulant (Wang et al. 2015a), anticancer (Konoshima and Takasaki 2002; Takasaki et al. 2003), antiobesity (Sun et al. 2012), anti-atherosclerotic (Takeo et al. 2002), hypocholesterolemic, hypolipidemic (Lin et al. 2007).

\section{Ziziphus jujuba Mill. RHAMNACEAE China}

\section{Da zao}

Fructus Ziziphi Jujubae Fruits [H453]

\section{Sweet. Warm. Stomach, spleen,} and heart meridians.

Stomach disorders, spleen deficiency: emaciation, weakness, dyspnea, diarrhea due to malnutrition, constipation, night sweating, blood deficiency: pale complexion, lips, and tongue, hypertension, skin aging, heart deficiency: palpitations, insomnia, anxiety, amnesia, depression, male sexual disorders: impotence.
Antioxidant, anti-inflammatory, neuroprotective: Alzheimer, gastro-intestinal protective, anticancer, cytotoxic, anti-obesity, hepatoprotective, immuno-enhancer, antifatigue (Bai et al. 2016; Gao et al. 2013; Lam et al. 2016b; Tahergorabi et al. 2015; ZareZardini et al. 2013; Zhang and Wu 2006), antidiabetic (Hemmati et al. 2015; Kaeidi et al. 2015), anti-constipation (Naftali et al. 2008), anti-dyslipidemic (Sabzghabaee et al. 2013), hematopoietic (Lam et al. 2016a), mnemonic (Heo et al. 2003), anti-insomnia (Yeung et al. 2012), sedative, anxiolytic, anti-allergic, contraceptive, hypotensive, antinephritic, antimicrobial, antiviral (Mahajan and Chopda 2009), antidepressant (Yeung et al. 2014a), sexual enhancer: erectile dysfunction (Chiou and Chen 2002). 
According to the results, 32 species of the TCP are marketed in the Barrio Chino supermarkets. Only for Nelumbo nucifera two distinct therapeutic products are discriminated: seeds and plumules; however, its uses linked to traditions and the biological activity overlap considerably, so these products were treated together. It is noteworthy that only three species are exclusively medicinal (Leonurus japonicus, Ligusticum striatus, and Prunella vulgaris), while the remaining 29 are also used for food, as vegetables, condiments, and sweeteners (Facciola 2001). In this regard, these species could be considered functional foods, i.e., foods that are consumed in the daily diet, and are a source of nutrients and important benefits to maintain health or reduce the risk of disease (Ferreira-Montero and Luengo-Fernández 2007; Hardy 2000).

From the viewpoint of the medicinal uses, of the total 32 species only eight $(25 \%)$ are considered within the denominated "50 fundamentals herbs" (Wong 1976) of the TCP: Astragalus mongholicus, Cinnamomum cassia, Eucommia ulmoides, Glycyrrhiza uralensis, Leonurus japonicus, Ligusticum striatum, Rehmannia glutinosa, and Schisandra chinensis. Also, 12 of the 32 treated species (37.5\%) are likewise used in Ayurveda medicine: Chrysanthemum morifolium, Cinnamomum cassia, Coix lacryma-jobi, Dioscorea oppositifolia, Fritillaria cirrhosa, Gastrodia elata, Glycyrrhiza uralensis, Houttuynia cordata, Kaempferia galangal, Ligusticum striatus, Nelumbo nucifera, and Prunella vulgaris (Khalsa and Tierra 2008; Khare 2008, 2011; Pole 2012).

The species used as anti-fatigue, antistress (adaptogenic), anti-aging, neuroprotective, and cognitive enhancer are: Angelica sinensis, Astragalus mongholicus, Atractylodes lancea, Codonopsis pilosula, Coix lacryma-jobi, Eleutherococcus senticosus, Eucommia ulmoides, Fallopia multiflora, Gastrodia elata, Glycyrrhiza uralensis, Houttuynia cordata, Kaempferia galanga, Leonurus japonicus, Lilium brownii, Lycium barbarum, Nelumbo nucifera, Ophiopogon japonicus, and Schisandra chinensis (18 species). The concept of "adaptogenic" has different meanings according to different authors; here it is used in its more restricted sense of anti-fatigue or anti-stress, easier to compare with the traditional uses (Hurrell et al. 2013, 2015a). The species used to treat sexual dysfunctions and genital sickness are: Angelica sinensis, Astragalus mongholicus, Cinnamomum cassia, Codonopsis pilosula, Eleutherococcus senticosus, Eucommia ulmoides, Fallopia multiflora, Gastrodia elata, Glycyrrhiza uralensis, Houttuynia cordata, Ligusticum striatum, Nelumbo nucifera, Paeonia lactiflora, Rehmannia glutinosa, Schisandra chinensis and Ziziphus jujuba (16 species). The species used for the respiratory system illnesses are: Chrysanthemum morifolium, Codonopsis pilosula, Dioscorea oppositifolia, Fritillaria cirrhosa, Glehnia littoralis, Glycyrrhiza uralensis, Houttuynia cordata, Kaempferia galanga, Lilium brownii, Lonicera japonica, Lycium barbarum, Ophiopogon japonicus, Scaphium affine, Schisandra chinensis, and Siraitia grosvenorii (15 species). The species mostly used to treat the digestive system disorders are: Amomum tsaoko, Atractylodes lancea, Cinnamomum cassia, Codonopsis pilosula, Coix lacryma-jobi, Glycyrrhiza uralensis, Kaempferia galanga, Lonicera japonica, Nelumbo nucifera, Ophiopogon japonicus, Paeonia lactiflora, Prunella vulgaris, Rehmannia glutinosa, Schisandra chinensis, and Ziziphus jujuba (15 species). For dermatological affections the species mostly used are: Astragalus mongholicus, Chrysanthemum morifolium, Cinnamomum cassia, Fallopia multiflora, Fritillaria cirrhosa, Glycyrrhiza uralensis, Houttuynia cordata, Kaempferia galanga, Leonurus japonicus, Lonicera japonica, Lycium barbarum, Prunella vulgaris, and Siraitia grosvenorii (13 species). The species used for the circulatory system disorders are: Angelica sinensis, Astragalus mongholicus, Cinnamomum cassia, Fritillaria cirrhosa, 
Glycyrrhiza uralensis, Leonurus japonicus, Ligusticum striatum, Lycium barbarum, Nelumbo nucifera, Rehmannia glutinosa, and Ziziphus jujuba (11 species). For osteo-arthro-muscular system affections the species used are: Angelica sinensis, Atractylodes lancea, Coix lacryma-jobi, Eleutherococcus senticosus, Eucommia ulmoides, Gastrodia elata, Glehnia littoralis, Houttuynia cordata, Ligusticum striatum, and Scaphium affine (10 species). The species used for the urinary system disorders are: Chrysanthemum morifolium, Cinnamomum cassia, Dioscorea oppositifolia, Eleutherococcus senticosus, Houttuynia cordata, Leonurus japonicus, Nelumbo nucifera, Rehmannia glutinosa, Schisandra chinensis (9 species).

The comparison between the columns of "Uses linked to traditions" and "Biological activity and effects evaluated" shows that mostly uses indicated in the first column have correlative studies listed in the second. Thus, it is possible to assert that the vast majority of uses awarded to the treated species have validation studies for the context of Western science. In the same way, it becomes evident that many effects evaluated exceed the list of uses linked to traditions. These effects generally include: anticancer, antioxidant, immunomodulatory or immuno-enhancer, anti-HIV, cardioprotective, anti-hypertensive, antiatherosclerotic, hypocholesterolemic, antihyperlipidemic, anti-obesity (slimming), antidiabetic, neuroprotective (Alzheimer, Parkinson), cognitive enhancer (memory and learning), anti-insomnia, anxiolytic, antidepressant, adaptogenic (anti-fatigue), and sexual enhancer (libido, frigidity, erectile dysfunction, and premature ejaculation). It is self-evident that these effects are evaluated because they respond to needs that are characteristic of health in the context of our Western societies, particularly, in our extensive metropolitan areas. In correlation, mass media do focus on this kind of conditions linked to the big cities lifestyle, and spread the scientific knowledge about the ailments, the plants that allow cure them, and the plant products accessible for the urban inhabitants. This complex dynamics in the transmission of botanical knowledge through their products in the urban scenario is reflected in many selling arguments, e.g. "plant products of a millenary tradition (Chinese) with scientifically studied effects". The consumer finds on these arguments two good reasons to select those products: tradition and science. The strategies selection operating on the information transmitted by the media are significant instances of the visualization process.

Heretofore, the visualization of Chinese plant products involves five species, about $15.6 \%$ of the total of surveyed species for the study area: Astragalus mongholicus, Chrysanthemum morifolium, Eleutherococcus senticosus, Lycium barbarum, and Schisandra chinensis. The other 27 treated species (84.4 $\%)$ still remain invisible to most of the local urban population. For the five visible species, the products that are sold in the Barrio Chino are also sold in the general commercial circuit. C. morifolium has only been found in one health food store of the general commercial circuit, so its visibility is scarce. By contrast, L. barbarum has been found in various outlets, and has wide diffusion in the Internet due to its many effects (adaptogenic, antioxidant, cognitive enhancer, hypocholesterolemic, antiatherosclerotic, sexual enhancer, anticancer, and antidiabetic, among others), so it is considered that its visibility is very wide. $A$. mongholicus, E. senticosus, and $S$. chinensis are also represented in the general commercial circuit through dietary supplements. These are capsules containing extracts of different species marketed specifically to treat the 
erectile dysfunction, widely disseminated through the media. In addition to its dissemination as a male sexual enhancer, these three species are usually valued by the same mentioned effects for $L$. barbarum.

The results obtained constitute the first inventory of species of medicinal plants introduced by Chinese immigrants in the study area. The inventory itself is a contribution of this work to the composition of the local botanical knowledge, through the plant products that commercialize this immigrants segment. It should be highlighted that this is the first time that an ethnobotanical work of this nature is carried out on Chinese immigrants in our country. Although this contribution is limited to the TCP plants, it is an important incentive for future works on other useful plants (especially food plants) introduced by the Chinese immigrants in the Buenos Aires Metropolitan Area.

In addition, the distinction between invisible and visible plant products for the majority of local urban inhabitants constitutes not only a conceptual distinction but also a methodological tool to address the study of the visualization process of invisible plant species that become visible: for the case of this contribution, 5 of 32 species surveyed. This is an original methodological tool developed in the research line on Urban Ethnobotany of the Laboratorio de Etnobotánica y Botánica Aplicada (LEBA) that allows evaluate the dynamics of the local botanical knowledge in relation to the circulation of the plant products in the local commercial circuits. Also, this methodological strategy has been satisfactorily tested in different contributions of the mentioned research line for the study area (i.e. Puentes and Hurrell 2015; Pochettino et al. 2012, for the segment of Bolivian immigrants). The value of the plant products and species inventory, and its associated knowledge, as well as the novel approach of applied methodology, constitutes an effort to provide adequate explanations for the complexity of the inherent phenomena in Urban Ethnobotany. Nevertheless, the results obtained and the discussion about them should not be considered a finished work but the current state of a dynamic process characteristic of pluricultural contexts.

\section{CONCLUSIONS}

From the above discussion it is clear that most of the TCP products surveyed in the Barrio Chino of Buenos Aires city still remain invisible to most of the local population, but its presence alone is significant because it increases the local diversity of useful plants, its products, and its associated knowledge. In sum, plant products that were entered through the Chinese immigrants segment into the local pluricultural context increase their biocultural diversity, i.e., the diversity of life, both in its biological dimension (species, plant products) and its cultural dimension (knowledge, beliefs, language, behaviors). In this framework, the biological and cultural dimensions are not separate: do not go on parallel tracks, on the contrary, these dimensions interact in complex ways and coevolve (Maffi 2001, 2005).

The visualization process, that involves the passage of plant products from the restricted circuit of immigrants to the general commercial circuit, is not only a mercantile movement but a communication process. It is necessary to remember that not only circulate the plant products (tangible or material component), together with these products circulate its associated knowledge (intangible component), i.e., the products work as messages (information source). In 
this context, from the circulation of the plant products emerges the information (for that matter, the botanical knowledge), enhanced by the mass media. In turn, that knowledge orients selective strategies of action about the plant products to consume. The entry of new plant products and its associated knowledge orients new strategies for consumption by local people that affect, to a greater or lesser extent, the whole botanical knowledge of the local pluricultural system. In terms of processes, new knowledge (new information) orients new strategies of action that act retroactively on the system of the local botanical knowledge, that is modified and adjusted to the new situations: it adapts, it evolves. In this way, plant products entering by the Chinese immigrants segment remain invisible and increase the biocultural diversity of the local pluricultural system, as noted above, or become visible and change the complex local system of knowledge and strategies of action, making possible its evolution.

The plants of Traditional Chinese Medicine in Buenos Aires city reveal the intrinsic complexity of the local botanical knowledge, especially by the contrast between Eastern traditions and the Western lifestyle of our large cities. However, visible Chinese species indicate that its incorporation to the local pluricultural mosaic is possible. The challenge ahead is to observe the becoming of Chinese plants that still remain invisible, and its role in the dynamic of changes of the local biocultural system.

\section{ACKNOWLEDGEMENTS}

The authors thank Dr. María Lelia Pochettino, Dr. Patricia M. Arenas, and the members of the Laboratorio de Etnobotánica y Botánica Aplicada (LEBA), for their continued collaboration, and the anonymous reviewers for their enriching comments. This research line is carried out with financial support of the Universidad Nacional de La Plata and the Consejo Nacional de Investigaciones Cientificas y Técnicas (CONICET), Argentina. 


\section{REFERENCES}

Achuthan CR, Padikkala J (1997) Hypolipidemic effect of Alpinia galanga (Rasna) and Kaempferia galanga (Kachoori). Indian Journal of Clinical Biochemistry 12:55-58

Adams JD, Lien EJ (2013) Traditional Chinese Medicine: Scientific basis for its use. RSC Drug Discovery Series, Cambridge, UK

Ahmed HM, Yeh J-Y, Tang Y-C, Cheng WT-K, Ou B-R (2014) Molecular screening of Chinese medicinal plants for progestogenic and antiprogestogenic activity. Journal of Biosciences 39:453-461

Ahn TS, Kim DG, Hong NR, Park HS, Kim H, Ha K-T, Jeon J-H, So I, Kim BJ (2015) Effects of Schisandra chinensis extract on gastrointestinal motility in mice. Journal of Ethnopharmacology 169:163-169

Ai L, Wu J, Che N, Wu Y, Cui SW (2012) Extraction, partial characterization and bioactivity of polysaccharides from boatfruited sterculia seeds. International Journal of Biological Macromolecules 51:815-818

Albuquerque UP, Cunha LVFC, Lucena RFP, Alves RRN (2014) Methods and Techniques in Ethnobiology and Ethnoecology. Springer New York, New York, USA

Albuquerque UP, Hurrell JA (2010) Ethnobotany: one concept and many interpretations. In: Albuquerque UP, Hanazaki N (eds) Recent Developments and Case Studies in Ethnobotany. SBEE/NUPEEA, Recife, Brazil, pp. 87-99

Amagase H, Nance DM (2011) Lycium barbarum Increases Caloric Expenditure and Decreases Waist Circumference in Healthy Overweight Men and Women: Pilot Study. Journal of the American College of Nutrition 30:304-309

Amagase $H$, Sun B, Nance DM (2009) Immunomodulatory effects of a standardized Lycium barbarum fruit juice in Chinese older healthy human subjects. Journal of Medicinal Food 12:1159-1165

An SM, Park CH, Heo JC, Park JY, Woo SU, Seo $\mathrm{JH}$, Lee MS, Cho KJ, Cho HS, Shin HM, Lee SH (2007) Gastrodia elata Blume protects against stress-induced gastric mucosal lesions in mice. International Journal of Molecular
Medicine 20:209-215

Aramwit P, Wirotsaengthong S (2013) Overview of commonly used Chinese herbs. Journal of Medicinal Plants Research 7:998-1014

Arenas PM, Doumecq B, Puentes JP, Hurrell JA (2015) Algas y plantas comercializadas como adelgazantes en el área metropolitana de Buenos Aires, Argentina. Gaia Scientia 9:32-40

Arentz S, Abbott JA, Smith CA, Bensoussan A (2014) Herbal medicine for the management of polycystic ovary syndrome (PCOS) and associated oligo/amenorrhoea and hyperandrogenism; a review of the laboratory evidence for effects with corroborative clinical findings. BMC Complementary and Alternative Medicine 14:511

Asea A, Kaur P, Panossian A, Wikman KG (2013) Evaluation of molecular chaperons Hsp72 and neuropeptide $Y$ as characteristic markers of adaptogenic activity of plant extracts. Phytomedicine 20:1323-1329

Ayeka PA, Bian Y, Mwitari PG, Chu X, Zhang Y, Uzayisenga R, Otachi EO (2016) Immunomodulatory and anticancer potential of Gan cao (Glycyrrhiza uralensis Fisch.) polysaccharides by CT-26 colon carcinoma cell growth inhibition and cytokine IL-7 upregulation in vitro. BMC Complementary and Alternative Medicine 16:206

Bai L, Zhang H, Liu Q, Zhao Y, Cui X, Guo S, Zhang L, Ho C-T, Bai N (2016) Chemical characterization of the main bioactive constituents from fruits of Ziziphus jujuba. Food Funct. 7:2870-2877

Barth A, Hovhannisyan A, Jamalyan K, Narimanyan M (2015) Antitussive effect of a fixed combination of Justicia adhatoda, Echinacea purpurea and Eleutherococcus senticosus extracts in patients with acute upper respiratory tract infection: A comparative, randomized, double-blind, placebo-controlled study. Phytomedicine 22:1195-1200

Bogado-Bordazar LL (2003) Migraciones Internacionales Influencia de la Migración China en Argentina y Uruguay. Universidad Nacional de La Plata, La Plata, Argentina.

Bokhari AA, Syed V (2015) Inhibition of Transforming Growth Factor- $\beta$ (TGF- $\beta$ ) Signaling by Scutellaria baicalensis and 
Fritillaria cirrhosa Extracts in Endometrial Cancer. Journal of Cellular Biochemistry 116:1797-1805

Bounda G-A, Feng Y (2015) Review of clinical studies of Polygonum multiflorum Thunb. and its isolated bioactive compounds. Pharmacognosy Research 7:225-236

Bradley RR, Cunniff PJ, Pereira BJG, Jaber BL (1999) Hematopoietic effect of Radix Angelicae sinensis in a hemodialysis patient. American Journal of Kidney Diseases 34:349-354

Cerrutti M (2009) Diagnóstico de las poblaciones de inmigrantes en la Argentina. Dirección Nacional de Población, Buenos Aires, Argentina.

Cha H-Y, Ahn S, Cheon J-H, Park I-S, Kim J-T, Kim K (2016) Hataedock Treatment Has Preventive Therapeutic Effects in Atopic Dermatitis-Induced NC/Nga Mice under HighFat Diet Conditions. Evidence-Based Complementary and Alternative Medicine 2016:1-13

Chan B-C, Hon K-L, Leung P-C, Sam S-W, Fung K-P, Lee M-Y, Lau H-Y (2008) Traditional Chinese medicine for atopic eczema: PentaHerbs formula suppresses inflammatory mediators release from mast cells. Journal of Ethnopharmacology 120:85-91

Chan S-W, Li P, Kwan Y-W, Lin G (2011) In vitro Tracheobronchial Relaxation of Fritillaria Alkaloids. Chinese Journal of Natural Medicines 9:345-353

Chan S-W, Li S-L, Lin G, Li P (2000) Pharmacokinetic Study and Determination of Imperialine, the Major Bioactive Component in Antitussive Fritillaria cirrhosa, in Rat by High-Performance Liquid Chromatography Coupled with Evaporative Light-Scattering Detector. Analytical Biochemistry 285:172-175

Chan YS, Ng TB (2013) A Lectin with Highly Potent Inhibitory Activity toward Breast Cancer Cells from Edible Tubers of Dioscorea opposita cv. Nagaimo. PLoS ONE 8:e54212

Chang H-M, But PP-H, Yao SC (2001) Pharmacology and Applications of Chinese Materia Medica. Vol 1. World Scientific Publishing Co. Pte. Ltd., Singapore

Chauhan A, Sharma K V, Chauhan S, Agarwal M (2009) Pharmacological Evaluation For The Antifertility Effect of the Ethanolic Seed
Extract of Nelumbo Nucifera (Sacred Lotus). Pharmacologyonline 2:636-643

Chen C-T, Wang Z-H, Hsu C-C, Lin H-H, Chen J$\mathrm{H}$ (2015) In Vivo Protective Effects of Diosgenin against Doxorubicin-Induced Cardiotoxicity. Nutrients 7:4938-4954

Chen H-H, Chiang W, Chang J-Y, Chien Y-L, Lee C-K, Liu K-J, Cheng Y-T, Chen T-F, Kuo Y-H, Kuo C-C (2011) Antimutagenic Constituents of Adlay (Coix lachryma-jobi L. var. ma-yuen Stapf) with Potential Cancer Chemopreventive Activity. Journal of Agricultural and Food Chemistry 59:6444-6452

Chen J, Liu JH, Wang T, Xiao HJ, Yin CP, Yang J (2008) Effects of plant extract neferine on cyclic adenosine monophosphate and cyclic guanosine monophosphate levels in rabbit corpus cavernosum in vitro. Asian Journal of Andrology 10:307-312.

Chen J, Wang Y, Hui C, Xi Y, Liu X, Qi F, Liu H, Wang Z, Niu S (2016a) Mechanisms of Heshouwuyin in regulating apoptosis of testicular cells in aging rats through mitochondrial pathway. BMC Complementary and Alternative Medicine 16:337

Chen M-H, Chen X-J, Wang M, Lin L-G, Wang YT (2016b) Ophiopogon japonicus. A phytochemical, ethnomedicinal and pharmacological review. Journal of Ethnopharmacology 181:193-213

Chen M-H, May BH, Zhou IW, Zhang AL, Xue CC (2016c) Integrative medicine for relief of nausea and vomiting in the treatment of colorectal cancer using oxaliplatin-based chemotherapy: A systematic review and meta-analysis. Phytotherapy Research 30:741-753

Chen P, Pang S, Yang N, Meng H, Liu J, Zhou N, Zhang M, Xu Z, Gao W, Chen B, Tao Z, Wang L, Yang $Z$ (2013) Beneficial Effects of Schisandrin $B$ on the Cardiac Function in Mice Model of Myocardial Infarction. PLoS ONE 8:e79418

Chen R, Moriya J, Yamakawa JI, Takahashi T, Kanda T (2010) Traditional chinese medicine for chronic fatigue syndrome. Evidence-Based Complementary and Alternative Medicine 7:3-10

Cheng JT, Lee YY, Hsu FL, Chang W, Niu CS (1944) Antihypertensive activity of phenolics from the flower of Lonicera japonica. Chinese Journal of Pharmacology 46:575-582 
Chiou W-F, Chen C-F (2002) Pharmacological profile of evodiamine in isolated rabbit corpus cavernosum. European Journal of Pharmacology 446:151-159

Choi G, Han A-R, Lee JH, Park J-Y, Kang U, Hong J, Kim YS, Seo E-K (2015) A Comparative Study on Hulled Adlay and Unhulled Adlay through Evaluation of Their LPS-Induced Anti-Inflammatory Effects, and Isolation of Pure Compounds. Chemistry \& Biodiversity 12:380-387

Choo MH, Jeong Y, Lee MY (2004) Effects of an Ethylacetate Fraction of Chrysanthemi Flos on the Antioxidative System and Lipid Profile in Rats with Ethanol-Induced Liver Damage. J Food Sci Nutr 9:352-360

Choo SH, Sung HH, Chae MR, Kang SJ, Han DH, Park JK, So I, Lee SW (2014) Effects of Schisandra chinensis extract on the relaxation of isolated human prostate tissue and smooth muscle cell. Journal of Ethnopharmacology 156:271-276

Chou S-T, Chang W-L, Chang C-T, Hsu S-L, Lin Y-C, Shih Y (2013) Cinnamomum cassia Essential Oil Inhibits $\alpha-M S H-I n d u c e d$ Melanin Production and Oxidative Stress in Murine B16 Melanoma Cells. International Journal of Molecular Sciences 14:19186-19201

Chun JN, Cho M, So I, Jeon J-H (2014) The protective effects of Schisandra chinensis fruit extract and its lignans against cardiovascular disease: $A$ review of the molecular mechanisms. Fitoterapia 97:224-233

Circosta C, Pasquale R De, Palumbo DR, Samperi S, Occhiuto F (2006) Estrogenic activity of standardized extract of Angelica sinensis. Phytotherapy Research 20:665-669

Collins NH, Lessey EC, DuSell CD, McDonnell DP, Fowler L, Palomino WA, Illera MJ, Yu X, Mo B, Houwing AM, Lessey BA (2009) Characterization of Antiestrogenic Activity of the Chinese Herb, Prunella vulgaris, Using In Vitro and In Vivo (Mouse Xenograft) Models. Biology of Reproduction 80:375-383

Corneanu M, Corneanu CG (2013) The Adaptogenic Species: Theoretical and Practical Importance. A Review. Oltenia 29:58-65

Cui Y, Wang X, Xue J, Liu J, Xie M (2014) Chrysanthemum morifolium extract attenuates high-fat milk-induced fatty liver through peroxisome proliferator-activated receptor $\alpha-$-mediated mechanism in mice. Nutrition Research 34:268-275

Dai M, Peng C, Peng F, Xie C, Wang P, Sun F (2015) Anti-Trichomonas vaginalis properties of the oil of Amomum tsao-ko and its major component, geraniol. Pharmaceutical Biology $54: 445-450$

Davydov M, Krikorian A (2000) Eleutherococcus senticosus Maxim. as an adaptogen: a closer look. Journal of Ethnopharmacology 72:345-393

De la Cruz JF, Vergara EJS, Cho Y, Hong HO, Oyungerel B, Hwang SG (2015) Glehnia littoralis Root Extract Induces G0/G1 Phase Cell Cycle Arrest in the MCF-7 Human Breast Cancer Cell Line. Asian Pacific Journal of Cancer Prevention 16:8113-8117

Debnath S, Kumar S, Bhattacharjee, Ganesh KG, Acharjee A, Vaddepally L (2011) A Review: Herbs used as sex stimulants. International Journal of Pharmaceutical Research and Technology 3:15-19

Doi K, Mitoma C, Nakahara T, Uchi H, Hashimoto-Hachiya A, Takahara M, Tsuji G, Nakahara M, Furue M (2014) Antioxidant Houttuynia cordata extract upregulates filaggrin expression in an aryl hydrocarbondependent manner. Fukuoka Igaku Zasshi 105:205-213

Drobnik J (2016) Chinese vegetative materia medica in a venereological treatise by Jean Astruc from 1740. Journal of Ethnopharmacology 187:293-301

Du M, Hu X, Kou L, Zhang B, Zhang C (2016) Lycium barbarum Polysaccharide Mediated the Antidiabetic and Antinephritic Effects in Diet-Streptozotocin-Induced Diabetic Sprague Dawley Rats via Regulation of NF- $K$ B. BioMed Research International 2016:1-9

Edewor-Kuponiyi TI (2013) Plant-Derived Compounds with Potential Sedative and Anxiolytic Activities. International Journal of Basic and Applied Science 2:63-78

Etkin NL, Ticktin T (2010) Advancing an ethnoecological perspective that integrates theory and method in ethnobotany. In: Albuquerque UP, Hanazaki N (eds) Recent Developments and Case Studies in Ethnobotany. SBEE/NUPEEA, Recife, Brazil, pp. 33-57

Facciola S (2001) Cornucopia II. A source 
book of edible plants. Kampong Publications, Vista, CA, USA

Fan Y, He Q, Luo A, Wang M, Luo A (2015) Characterization and Antihyperglycemic Activity of a Polysaccharide from Dioscorea opposita Thunb Roots. International Journal of Molecular Sciences 16:6391-6401

Farahani MS, Bahramsoltani R, Farzaei $M H$, Abdollahi M, Rahimi R (2015) Plant-derived natural medicines for the management of depression: an overview of mechanisms of action. Reviews in the Neurosciences 26:305-321

Feng X-T, Qin C-B, Leng J, Tang Q-L, Shi H, Zhai L-N, Li S-L (2012) Yidiyin, a Chinese Herbal Decoction, Improves Erectile Dysfunction in Diabetic Patients and Rats through the NOcGMP Pathway. Bioscience, Biotechnology, and Biochemistry 76:257-263

Feng X, Jiang Z, Li R (2009) Research progress in essential oil of natural food spice, Amomum tsao-ko. China Condiment 8:40-42

Ferreira-Montero IJ, Luengo-Fernández E (2007) La dieta como concepto terapéutico. Conceptos de alimento funcional $y$ de nutracéutico. Situación actual de los alimentos funcionales y nutracéuticos. Aspectos legales. In: Luengo-Fernández E (ed) Alimentos funcionales y nutracéuticos. Sociedad Española de Cardiología, Madrid, España, pp. 1-12

Fleischer T, Chang T, Yen H (2016) Posthematopoietic stem cell transplantation in patients with hematologic disorders: Chinese herbal medicine for an unmet need. Journal of Integrative Medicine 14:322-335

Forstall RL, Greene RP, Pick JB (2009) Which are the Largest? Why Lists of Major Urban Areas Vary so Greatly. Tijdschrift voor economische en sociale geografie 100:277-297

Foster S, Yue C (1992) Herbal emissaries. Bringing Chinese herbs to the West. Healing Arts Press, Rochester, VT, USA

Fu J, Dai L, Lin Z, Lu H (2013) Houttuynia cordata Thunb: A Review of Phytochemistry and Pharmacology and Quality Control. Chinese Medicine 4:101-123

Fu K, Piao T, Wang M, Zhang J, Jiang J, Wang X, Liu H (2014) Protective effect of catalpol on lipopolysaccharide-induced acute lung injury in mice. International Immunopharmacology

\section{3:400-406}

Fu X, Wang Q, Wang Z, Kuang H, Jiang P (2015)

Danggui-Shaoyao-San: New Hope for Alzheimer's Disease. Aging and Disease 7:502-513

Fukada K, Kajiya-Sawane M, Matsumoto $\mathrm{Y}$, Hasegawa T, Fukaya Y, Kajiya K (2016) Antiedema effects of Siberian ginseng in humans and its molecular mechanism of lymphatic vascular function in vitro. Nutrition Research 36:689-695

Gairola S, Gupta V, Bansal P, Singh R, Maithani $M(2010)$ Herbal antitussive and expectorants. A review. International Journal of Pharmaceutical Sciences Review and Research 5:5-9

Gao M, Zhang JH, X ZF, H XC, G H, Q FS, F ZY (2012a) Angelica sinensis suppresses human lung adenocarcinoma $A 549$ cell metastasis by regulating MMPs/TIMPs and TGF- $\beta 1$. Oncology Reports 27:585-593

Gao Q-H, Wu C-S, Wang M (2013) The Jujube (Ziziphus jujuba Mill.) Fruit: A Review of Current Knowledge of Fruit Composition and Health Benefits. Journal of Agricultural and Food Chemistry 61:3351-3363

Gao S, Wang H, Zeng C, Hou J, Zhang Y (2012b) Phytochemical and Pharmacological Properties of Radix Codonopsis: A Review. Journal of Chinese Medicine Research and Development 1:16-22

Goswami SK, Inamdar MN, Jamwal R, Dethe S (2013) Efficacy of Cinnamomum cassia Blume. in age induced sexual dysfunction of rats. Journal of Young Pharmacists 5:148-153

Goswami SK, Inamdar MN, Jamwal R, Dethe S (2014) Effect of Cinnamomum cassia Methanol Extract and Sildenafil on Arginase and Sexual Function of Young Male Wistar Rats. The Journal of Sexual Medicine 11:1475-1483

Gui M, Du J, Guo J, Xiao B, Yang W, Li M (2014) Aqueous Extract of Chrysanthemum morifolium Enhances the Antimelanogenic and Antioxidative Activities of the Mixture of Soy Peptide and Collagen Peptide. Journal of Traditional and Complementary Medicine 4:171-176

Guo A, Choi R, Zheng K, Chen V, Dong T, Wang Z-T, Vollmer G, Lau D, Tsim K (2012) Kaempferol as a flavonoid induces 
osteoblastic differentiation via estrogen receptor signaling. Chinese Medicine 7:10

Haarberg KM, Wymore BMJ, Overstreet AM, Hauck CC, Murphy PA, Hostetter JM, Ramer-Tait AE, Wannemuehler MJ (2015) Orally administered extract from Prunella vulgaris attenuates spontaneous colitis in mdr1a- $/$ mice. World Journal of Gastrointestinal Pharmacology and Therapeutics 6:223-237

Han C, Guo J (2012) Antibacterial and Antiinflammatory Activity of Traditional Chinese Herb Pairs, Angelica sinensis and Sophora flavescens. Inflammation 35:913-919

Han DH, Lee JH, Kim H, Ko MK, Chae MR, Kim HK, So I, Jeon J-H, Park JK, Lee SW (2012) Effects of Schisandra chinensis extract on the contractility of corpus cavernosal smooth muscle (CSM) and Ca2+ homeostasis in CSM cells. BJU International 109:1404-1413

Han K, Meng W, Zhang J-J, Zhou Y, Wang Y, Su Y, Lin S, Gan Z, Sun Y, Min D-L (2016) Luteolin inhibited proliferation and induced apoptosis of prostate cancer cells through miR-301. OncoTargets and Therapy 9:3085-3094

Han LQ, Dong SF, Liu JH (2008) Determination of the contents of trace elements in Chinese herbal medicines for treating respiratory system diseases. Guang Pu Xue Yu Guang Pu Fen Xi 28:453-455

Han M-N, Lu J-M, Zhang G-Y, Yu J, Zhao R-H (2015) Mechanistic Studies on the Use of Polygonum multiflorum for the Treatment of Hair Graying. BioMed Research International 2015:1-8

Han Y, Jung HW, Bae HS, Kang J-S, Park Y-K (2013) The extract of Cinnamomum cassia twigs inhibits adipocyte differentiation via activation of the insulin signaling pathway in 3T3-L1 preadipocytes. Pharmaceutical Biology 51:961-967

Hardy G (2000) Nutraceuticals and functional foods: introduction and meaning. Nutrition 16:698-699

Hazard-Vallerand A, Sanoski CA, Hopfer-Deglin J (2016) Davis's Drug Guide for Nurses. 15th ed ed. Davis Co. Philadelphia, USA

He CL, Yang XH, Huang H, Yang QZ (2009) Anti-weary pharmacological action of Lily polysaccharide. Journal of Hunan Normal University (Medical Sciences) 3:9-11, 15
He D-Y, Dai S-M (2011) Anti-Inflammatory and Immunomodulatory Effects of Paeonia lactiflora Pall., a Traditional Chinese Herbal Medicine. Frontiers in Pharmacology 2:

He K, Li X, Chen X, Ye X, Huang J, Jin Y, Li P, Deng Y, Jin Q, Shi Q, Shu H (2011) Evaluation of antidiabetic potential of selected traditional Chinese medicines in STZ-induced diabetic mice. Journal of Ethnopharmacology 137:1135-1142

He X, Wang J, Li M, Hao D, Yang Y, Zhang C, He R, Tao R (2014) Eucommia ulmoides Oliv.: Ethnopharmacology, phytochemistry and pharmacology of an important traditional Chinese medicine. Journal of Ethnopharmacology 151:78-92

He Z, Wang D, Shi L, Wang L (1986) Treating amenorrhea in vital energy-deficient patients with Angelica sinensis-Astragalus membranaceus menstruation-regulating decoction. Journal of Traditional Chinese Medicine 6:187-190

Hehir MP, Morrison JJ (2016) Paeoniflorin, a novel heat-shock protein inducing compound, and human myometrial contractility in vitro. Journal of Obstetrics and Gynaecology Research 42:302-306

Hemmati M, Asghari S, Zohoori E, Karamian M (2015) Hypoglycemic effects of three Iranian edible plants; Jujube, barberry and saffron: Correlation with serum adiponectin level. Pakistan Journal of Pharmaceutical Sciences 28:2095-2099

Hempen CH, Fischer T (2009) A Materia Medica for Chinese Medicine: Plants, Minerals, and Animal Products. Churchill Livingstone Elsevier, Munich, Germany

Heo H-J, Park Y-J, Suh Y-M, Choi S-J, Kim M-J, Cho H-Y, Chang Y-J, Hong B, KIM H-K, Kim E, Kim C-J, Kim B-G, Shin D-H (2003) Effects of Oleamide on Choline Acetyltransferase and Cognitive Activities. Bioscience, Biotechnology, and Biochemistry 67:1284-1291

Hetchman L (2013) Clinical naturopathic medicine. Churchill Livingstone Elsevier, Sydney, Australia

Hong SH, Kwon JT, Shin JY, Kim JE, MinaiTehrani A, Yu KN, Lee S, Park SJ, Chang SH, Jiang $\mathrm{HL}$, Vibin M, Han K, Son K, Kwak WJ, Chae C, Bang SH, Cho MH (2013) Therapeutic effect of Broussonetia papyrifera and 
Lonicera japonica in ovalbumin-induced murine asthma model. Natural Product Communications 8:1609-1614

Hou JP, Jin Y (2005) The Healing Power of Chinese Herbs and Medicinal Recipes. The Haworth Integrative Healing Press, New York, USA

Hsieh M-T, Peng W-H, Wu C-R, Wang W-H (2000) The ameliorating effects of the cognitive-enhancing Chinese herbs on scopolamine-induced amnesia in rats. Phytotherapy Research 14:375-377

Hsu C-H, Lin C-L, Wang S-E, Sheu S-J, Chien C$\mathrm{T}$, Wu C-H (2015) Oral treatment with herbal formula B401 alleviates penile toxicity in aging mice with manganism. Clinical Interventions in Aging 10:907-918

Hsu C-S, Yang J-K, Yang L-L (2006) Effect of "Dang-Qui-Shao-Yao-San" a Chinese medicinal prescription for dysmenorrhea on uterus contractility in vitro. Phytomedicine 13:94-100

Hsu P-C, Tsai Y-T, Lai J-N, Wu C-T, Lin S-K, Huang C-Y (2014) Integrating traditional Chinese medicine healthcare into diabetes care by reducing the risk of developing kidney failure among type 2 diabetic patients: A population-based case control study. Journal of Ethnopharmacology 156:358-364

Hu Y, Bi X, Zhao P, Zheng H, Huang X (2015) Cytotoxic Activities, SAR and Anti-Invasion Effects of Butylphthalide Derivatives on Human Hepatocellular Carcinoma SMMC7721 Cells. Molecules 20:20312-20319

Huang C-H, Liu D-Z, Jan T-R (2010) Diosgenin, a Plant-Derived Sapogenin, Enhances Regulatory T-Cell Immunity in the Intestine of Mice with Food Allergy. Journal of Natural Products 73:1033-1037

Huang L, Zhao H, Huang B, Zheng C, Peng W, Qin L (2011) Acanthopanax senticosus: Review of botany, chemistry and pharmacology. Pharmazie 66:83-97

Huang Q, Gao B, Wang L, Zhang HY, Li XJ, Shi J, Wang Z, Zhang JK, Yang L, Luo ZJ, Liu J (2015a) Ophiopogonin D: A new herbal agent against osteoporosis. Bone 74:18-28

Huang Q, Shi J, Gao B, Zhang HY, Fan J, Li XJ, Fan JZ, Han YH, Zhang JK, Yang L, Luo ZJ, Liu $J$ (2015b) Gastrodin: an ancient Chinese herbal medicine as a source for anti- osteoporosis agents via reducing reactive oxygen species. Bone 73:132-144

Huang $Y$, Jiang $C$, Hu Y, Zhao X, Shi C, Yu Y, Liu $\mathrm{C}$, Tao $\mathrm{Y}$, Pan $\mathrm{H}$, Feng $\mathrm{Y}$, Liu J, Wu Y, Wang D (2013) Immunoenhancement effect of Rehmannia glutinosa polysaccharide on lymphocyte proliferation and dendritic cell. Carbohydrate Polymers 96:516-521

Huh E, Kim HG, Park H, Kang MS, Lee B, Oh MS (2014) Houttuynia cordata Improves Cognitive Deficits in Cholinergic Dysfunction Alzheimer's Disease-Like Models. Biomolecules \& Therapeutics 22:176-183

Huh J-E, Lee W-I, Seo B-K, Baek Y-H, Lee J-D, Choi D-Y, Park D-S (2011) Gastroprotective and safety effects of WIN-34B, a novel treatment for osteoarthritis, compared to NSAIDs. Journal of Ethnopharmacology 137:1011-1017

Huh J-E, Seo B-K, Baek Y-H, Lee S, Lee J-D, Choi D-Y, Park D-S (2012) Standardized butanol fraction of WIN-34B suppresses cartilage destruction via inhibited production of matrix metalloproteinase and inflammatory mediator in osteoarthritis human cartilage explants culture and chondrocytes. BMC Complementary and Alternative Medicine 12:1245

Hung Y-C, Kao C-W, Lin C-C, Liao Y-N, Wu B-Y, Hung I-L, Hu W-L (2016) Chinese Herbal Products for Female Infertility in Taiwan. A Population-Based Cohort Study. Medicine 95:e3075

Hurrell JA (2014a) Urban Ethnobotany in Argentina: Theoretical advances and methodological strategies. Ethnobiology and Conservation 3:2

Hurrell JA (2014b) Dinámica del conocimiento botánico local: plantas de la Fitoterapia Tradicional China comercializadas en la ciudad de Buenos Aires (Argentina). Actas ICEB 2014, Córdoba, España

Hurrell JA, Arenas PM, Cristina I (2015a) EI conocimiento botánico en zonas urbanas: potenciadores cognitivos comercializados en el área metropolitana de Buenos Aires, Argentina. Gaia Scientia 9:17-31

Hurrell JA, Pochettino ML (2014) Urban Ethnobotany: theoretical and methodological contributions. In: Albuquerque UP, Cruz LVFC, Lucena RFP, Alves RRN (eds) Methods and 
Techniques in Ethnobiology and Ethnoecology. Springer New York, New York, USA, pp. 293-309

Hurrell JA, Pochettino ML, Puentes JP, Arenas PM (2013) Del marco tradicional al escenario urbano: Plantas ancestrales devenidas suplementos dietéticos en la conurbación Buenos Aires-La Plata, Argentina. Boletín Latinoamericano y del Caribe de Plantas Medicinales y Aromáticas 12:499-515

Hurrell JA, Puentes JP (2013) Medicinal and aromatic species of Asteraceae commercialized in the conurbation Buenos Aires-La Plata (Argentina). Ethnobiology and Conservation 2:7

Hurrell JA, Puentes JP (2015) Nuevos registros de productos de la Fitoterapia Tradicional China comercializados en la ciudad de Buenos Aires, Argentina. Boletín de la Sociedad Argentina de Botánica 50 (Supl):150.

Hurrell JA, Puentes JP, Arenas PM (2015b) Medicinal plants with cholesterol-lowering effect marketed in the Buenos Aires-La Plata conurbation, Argentina: An urban ethnobotany study. Ethnobiology and Conservation 4:7

Hurrell JA, Puentes JP, Arenas PM (2016) Estudios etnobotánicos en la conurbación Buenos Aires-La Plata, Argentina: productos de plantas medicinales introducidos por inmigrantes paraguayos. Bonplandia 25:43-52

Hussain T, Tan B, Liu G, Oladele OA, Rahu N, Tossou MC, Yin $Y$ (2016) Health-Promoting Properties of Eucommia ulmoides : A Review. Evidence-Based Complementary and Alternative Medicine 2016:1-9

Hwang SM, Kim JS, Lee YJ, Yoon JJ, Lee SM, Kang DG, Lee HS (2012) Anti-Diabetic Atherosclerosis Effect of Prunella vulgaris in $\mathrm{db} / \mathrm{db}$ Mice with Type 2 Diabetes. The American Journal of Chinese Medicine 40:937-951

INDEC (2016) Instituto Nacional de Estadística y Censos. Censo 2010. [http://www.indec.gob.ar/] Accessed December 20, 2016

Ishikawa T, Swga Y, Kitajima J (2001) WaterSoluble Constituents of Glehnia littoralis Fruit. Chemical \& Pharmaceutical Bulletin 49:584-588

Jang YW, Lee JY, Kim CJ (2010) Anti-asthmatic activity of phenolic compounds from the roots of Gastrodia elata BI. International Immunopharmacology 10:147-154

Jeon S, Lee C-H, Liu QF, Kim GW, Koo B-S, Pak SC (2014) Alteration in brain-derived neurotrophic factor (BDNF) after treatment of mice with herbal mixture containing Euphoria longana, Houttuynia cordata and Dioscorea japonica. DARU Journal of Pharmaceutical Sciences 22:77

Jeon WK, Kim YE, Park SO, Kwon DY, Ahn SW, Lee JH, Ji MS, Ko BS (2008) The modified Je-Ho-Tang, Korean herbal medicine, inhibits whole-blood aggregation and platelet adhesion to collagen under flow. Thrombosis Research 122:804-809

Jeong EJ, Jegal J, Ahn J, Kim J, Yang MH (2016) Anti-obesity Effect of Dioscorea oppositifolia Extract in High-Fat Diet-Induced Obese Mice and Its Chemical Characterization. Biological \& Pharmaceutical Bulletin 39:409-414

Ji S, Li Z, Song W, Wang Y, Liang W, Li K, Tang S, Wang Q, Qiao X, Zhou D, Yu S, Ye M (2016) Bioactive Constituents of Glycyrrhiza uralensis (Licorice): Discovery of the Effective Components of a Traditional Herbal Medicine. Journal of Natural Products 79:281-292

Jia W, Gao W, Tang L (2003) Antidiabetic herbal drugs officially approved in China. Phytotherapy Research 17:1127-1134

Jiang J, Li Y, Feng Z, Yang Y, Zhang P (2015) Glucaric acids from Leonurus japonicus. Fitoterapia 107:85-89

Jin D, Cao M, Mu X, Yang G, Xue W, Huang Y, Chen H (2015) Catalpol Inhibited the Proliferation of T24 Human Bladder Cancer Cells by Inducing Apoptosis Through the Blockade of Akt-Mediated Anti-apoptotic Signaling. Cell Biochemistry and Biophysics 71:1349-1356

Jin J-S, Lee J-H (2012) Phytochemical and pharmacological aspects of Siraitia grosvenorii, luo han kuo. Oriental Pharmacy and Experimental Medicine 12:233-239

Jin M, Huang Q, Zhao K, Shang P (2013) Biological activities and potential health benefit effects of polysaccharides isolated from Lycium barbarum L. International Journal of Biological Macromolecules 54:16-23

Jin M, Zhao K, Huang Q, Shang P (2014) Structural features and biological activities of 
the polysaccharides from Astragalus membranaceus. International Journal of Biological Macromolecules 64:257-266

Ju Y, Xue Y, Huang J, Zhai Q, Wang X (2014) Antioxidant Chinese yam polysaccharides and its pro-proliferative effect on endometrial epithelial cells. International Journal of Biological Macromolecules 66:81-85

Jung HA, Karki S, Kim JH, Choi JS (2015) BACE1 and cholinesterase inhibitory activities of Nelumbo nucifera embryos. Archives of Pharmacal Research 38:1178-1187

Jung $\mathrm{Y}-\mathrm{H}$, Kwon S-H, Hong S-I, Lee S-O, Kim SY, Lee S-Y, Jang C-G (2012) 5-HT1A receptor binding in the dorsal raphe nucleus is implicated in the anxiolytic-like effects of Cinnamomum cassia. Pharmacology Biochemistry and Behavior 103:367-372

Jung YB, Roh K-J, Jung J-A, Jung K, Yoo H, Cho Y-B, Kwak W-J, Kim D-K, Kim KH, Han C-K (2001) Effect of SKI 306X, a New Herbal AntiArthritic Agent, in Patients with Osteoarthritis of the Knee: A Double-Blind Placebo Controlled Study. The American Journal of Chinese Medicine 29:485-491

Jung Koo H, Sohn E-H, Kim Y-J, Jang S-A, Namkoong S, Chan Kang S (2014) Effect of the combinatory mixture of Rubus coreanus Miquel and Astragalus membranaceus Bunge extracts on ovariectomy-induced osteoporosis in mice and anti-RANK signaling effect. Journal of Ethnopharmacology 151:951-959

Kaeidi A, Taati M, Hajializadeh Z, Jahandari F, Rashidipour M (2015) Aqueous extract of Zizyphus jujuba fruit attenuates glucose induced neurotoxicity in an in vitro model of diabetic neuropathy. Iranian Journal of Basic Medical Sciences 18:301-306

Kakatum N, Jaiarree N, Makchucit S, Itharat A (2012) Antioxidant and anti-inflammatory activities of Thai medicinal plants in Sahasthara remedy for muscle pain treatment. Journal of the Medical Association of Thailand 95:120-126

Kang C, Lee H, Hah DY, Heo JH, Kim CH, Kim E, Kim JS (2013a) Protective effects of Houttuynia cordata Thunb. on Gentamicininduced oxidative stress and nephrotoxicity in rats. Toxicological Research 29:61-67.

Kang JW, Nam DW, Kim KH, Huh JE, Lee JD (2013b) Effect of Gambisan on the inhibition of adipogenesis in 3T3-L1 adipocytes. Evidence-Based Complementary and Alternative Medicine 2013:1-11

Kato E, Inagaki Y, Kawabata J (2015a) Higenamine 4'-O- $\beta-d-g l u c o s i d e$ in the lotus plumule induces glucose uptake of L6 cells through $\boldsymbol{\beta} 2$-adrenergic receptor. Bioorganic \& Medicinal Chemistry 23:3317-3321

Kato S, Kato Y, Shibata H, Saitoh Y, Miwa N (2015b) Repressive effects of oat extracts on intracellular lipid-droplet formation in adipocytes and a three-dimensional subcutaneous adipose tissue model. Materials Science and Engineering C 49:269-273

Kato T, Horie N, Matsuta T, Naoki U, Shimoyama T, Kaneko T, Kanamoto T, Terakubo S, Nakashima H, Kusama K, Sakagami H (2012) Anti-UV/HIV activity of Kampo medicines and constituent plant extracts. In Vivo 26:1007-1014

Kavandi L, Lee LR, Bokhari AA, Pirog JE, Jiang Y, Ahmad KA, Syed V (2015) The Chinese herbs Scutellaria baicalensis and Fritillaria cirrhosa target NFKB to inhibit proliferation of ovarian and endometrial cancer cells. Molecular Carcinogenesis 54:368-378

Khalsa KPS, Tierra M (2008) The Way of Ayurvedic Herbs. Lotus Press, Twin Lakes, WI, USA.

Khan S, Shehzad O, Jin H-G, Woo E-R, Kang SS, Baek SW, Kim J, Kim YS (2012) Antiinflammatory Mechanism of 15,16-Epoxy-3ahydroxylabda-8,13(16),14-trien-7-one via Inhibition of LPS-Induced Multicellular Signaling Pathways. Journal of Natural Products 75:67-71

Khare CP (2008) Indian Medicinal Plants: An Illustrated Dictionary. Springer Science + Business Media, LLC, Berlin, Germany

Khare CP (2011) Indian Herbal Remedies. Springer, Berlin, Germany

Kim E-C, Kim HJ, Kim T-J (2015a) Water extract of Cinnamomum cassia suppresses angiogenesis through inhibition of VEGF receptor 2 phosphorylation. Bioscience, Biotechnology, and Biochemistry 79:617-624

Kim ES, Weon JB, Yun B-R, Lee J, Eom MR, Oh $\mathrm{K}-\mathrm{H}$, Ma CJ (2014) Cognitive Enhancing and Neuroprotective Effect of the Embryo of the Nelumbo nucifera Seed. Evidence-Based 
Complementary and Alternative Medicine 2014:1-9

Kim HG, Jeong HU, Hong SI, Oh MS (2015b) Houttuyniae Herba attenuates kainic acidinduced neurotoxicity via calcium response modulation in the mouse hippocampus. Planta Medica 81:1697-1704.

Kim HJ, Seo J-Y, Suh H-J, Lim SS, Kim J-S (2012) Antioxidant activities of licoricederived prenylflavonoids. Nutrition Research and Practice 6:491-498

Kim S-J, Kim J-S, Choi H-S, Kim Y-M, Hong S-W, Hum Yeon S, Kim Y, Lee S-M (2015c) HS-23, a Lonicera japonica extract, reverses sepsisinduced immunosuppression by inhibiting lymphocyte apoptosis. Journal of Ethnopharmacology 171:231-239

Kim SY, Koo YK, Koo JY, Ngoc TM, Kang SS, Bae K, Kim YS, Yun-Choi HS (2010) Platelet Anti-Aggregation Activities of Compounds from Cinnamomum cassia. Journal of Medicinal Food 13:1069-1074

Knipping K, Garssen J, van't Land B (2012) An evaluation of the inhibitory effects against rotavirus infection of edible plant extracts. Virology Journal 9:137

Ko HJ, Kwon OS, Jin JH, Son KH, Kim HP (2013) Inhibition of Experimental Systemic Inflammation (Septic Inflammation) and Chronic Bronchitis by New Phytoformula BL Containing Broussonetia papyrifera and Lonicera japonica. Biomolecules and Therapeutics 21:66-71

Kobayashi Y, Watanabe M, Ogihara J, Kato J, Oishi K (2000) Inhibition of HIV-1 Reverse Transcriptase by Methanol Extracts of Commercial Herbs and Spices. Nippon Shokuhin Kagaku Kogaku Kaishi 47:642-645

Kong C-S, Um YR, Lee JI, Kim YA, Yea SS, Seo $Y$ (2010) Constituents isolated from Glehnia littoralis suppress proliferations of human cancer cells and MMP expression in HT1080 cells. Food Chemistry 120:385-394

Konoshima T, Takasaki M (2002) Cancerchemopreventive effects of natural sweeteners and related compounds. Pure and Applied Chemistry 74:1309-1316

Kook M, Lee SK, Kim SD, Lee HY, Hwang JS, Choi YW, Bae YS (2015) Anti-septic activity of a-cubebenoate isolated from Schisandra chinensis. BMB Reports 48:336-341
Koonrungsesomboon N, Na-Bangchang K, Karbwang J (2014) Therapeutic potential and pharmacological activities of Atractylodes lancea (Thunb.) DC. Asian Pacific Journal of Tropical Medicine 7:421-428

Kou J, Tian Y, Tang Y, Yan J, Yu B (2006) Antithrombotic Activities of Aqueous Extract from Radix Ophiopogon japonicus and Its Two Constituents. Biological \& Pharmaceutical Bulletin 29:1267-1270

Kuchta K, Ortwein J, Rauwald HW (2012) Leonurus japonicus, Leonurus cardiaca, Leonotis leonurus: A novel HPLC study on the occurrence and content of the pharmacologically active guanidino derivative leonurine. Die Pharmazie - An International Journal of Pharmaceutical Sciences 67:973-979

Kumar A (2014) Chemical composition of essential oil isolated from the rhizomes of Kaempferia galanga L. International Journal of Pharma and Bio Sciences 5:225-231

Kumar M, Prasad SK, Hemalatha S (2014a) A current update on the phytopharmacological aspects of Houttuynia cordata Thunb. Pharmacognosy Reviews 8:22-35

Kumar M, Prasad SK, Krishnamurthy S, Hemalatha S (2014b) Antihyperglycemic Activity of Houttuynia cordata Thunb. in Streptozotocin-Induced Diabetic Rats. Advances in Pharmacological Sciences 2014:1-12

Kumar T, Chaiyasut C, Rungseevijitprapa W, Suttajit M (2011) Screening of steroid 5reductase inhibitory activity and total phenolic content of Thai plants. Journal of Medicinal Plants Research 5:1265-1271

Kurokawa M, Kumeda CA, Yamamura J, Kamiyama T, Shiraki K (1998) Antipyretic activity of cinnamyl derivatives and related compounds in influenza virus-infected mice. European Journal of Pharmacology 348:45-51

Kwan C-Y, Zhang W-B, Sim S-M, Deyama T, Nishibe S (2004) Vascular effects of Siberian ginseng (Eleutherococcus senticosus): endothelium-dependent NO- and EDHFmediated relaxation depending on vessel size. Naunyn-Schmiedeberg's Archives of Pharmacology 369:473-480

Kwon G, Kim HJ, Park SJ, Lee HE, Woo H, Ahn YJ, Gao Q, Cheong JH, Jang DS, Ryu JH (2014) Anxiolytic-like effect of danshensu [(3-(3,4- 
dihydroxyphenyl)-lactic acid)] in mice. Life Sciences 101:73-78

Ladio AH, Albuquerque UP (2016) Urban Ethnobiology. In: Albuquerque UP, Alves RRN (eds) Introduction to Ethnobiology. Springer, New York, USA pp. 33-38

Ladio AH, Albuquerque UP (2014) The concept of hybridization and its contribution to urban ethnobiology. Ethnobiology and Conservation 3:6

Lam CTW, Chan PH, Lee PSC, Lau KM, Kong AYY, Gong AGW, Xu ML, Lam KYC, Dong TTX, Lin H, Tsim KWK (2016a) Chemical and biological assessment of Jujube (Ziziphus jujuba)-containing herbal decoctions: Induction of erythropoietin expression in cultures. Journal of Chromatography Bioanalysis of Traditional Chinese Medicines 1026:254-262

Lam CTW, Gong AGW, Lam KYC, Zhang LM, Chen J-P, Dong TTX, Lin H-Q, Tsim KWK (2016b) Jujube-containing herbal decoctions induce neuronal differentiation and the expression of anti-oxidant enzymes in cultured PC12 cells. Journal of Ethnopharmacology 188:275-283

Lam TL, Lam ML, Au TK, Ip DTM, Ng TB, Fong WP, Wan DCC (2000) A comparison of human immunodeficiency virus type-1 protease inhibition activities by the aqueous and methanol extracts of Chinese medicinal herbs. Life Sciences 67:2889-2896

Lam W, Jiang Z, Guan F, Huang X, Hu R, Wang J, Bussom S, Liu S-H, Zhao H, Yen Y, Cheng Y-C (2015) PHY906(KD018), an adjuvant based on a 1800-year-old Chinese medicine, enhanced the anti-tumor activity of Sorafenib by changing the tumor microenvironment. Scientific Reports 5:9384

Lee B-J, Kim Y-J, Cho D-H, Sohn N-W, Kang H (2011) Immunomodulatory effect of water extract of cinnamon on anti-CD3-induced cytokine responses and p38, JNK, ERK1/2, and STAT4 activation. Immunopharmacology and Immunotoxicology 33:714-722

Lee G-H, Lee M-R, Lee H-Y, Kim SH, Kim H-K, Kim H-R, Chae H-J (2014) Eucommia ulmoides Cortex, Geniposide and Aucubin Regulate Lipotoxicity through the Inhibition of Lysosomal BAX. PLoS ONE 9:e88017

Lee IS, Kim KS, Kim KH, Park J, Jeong HS, Kim $\mathrm{Y}, \mathrm{Na} Y \mathrm{C}$, Lee SG, Ahn KS, Lee JH, Jang HJ (2016a) Anti-diabetic and anti-obesitic effects of aqueous extracts of Yangkyuksanhwa-tang and its two major compositions on $\mathrm{db} / \mathrm{db}$ mice. Biomedicine \& Pharmacotherapy 83:431-438

Lee JE, Kim MH, Choi YY, Lee HJ, Yang WM (2016b) Regulation of osteoclastic and osteoblastic differentiation marker expressions in osteoblast-like saos-2 cells by Eucommia ulmoides. Oriental Pharmacy and Experimental Medicine 16:53-57

Lee K-H, Choi E-M (2006) Stimulatory effects of extract prepared from the bark of Cinnamomum cassia blume on the function of osteoblastic MC3T3-E1 cells. Phytotherapy Research 20:952-960

Lee K-P, Kang S, Park S-J, Kim J-M, Lee J-M, Lee A-Y, Chung H-Y, Choi Y-W, Lee Y-G, Im D-S (2015a) Anti-allergic effect of $\alpha$-cubebenoate isolated from Schisandra chinensis using in vivo and in vitro experiments. Journal of Ethnopharmacology 173:361-369

Lee $Y$, Choi H, Seo M, Jeon H, Kim K, Lee B (2015b) Kaempferol suppresses lipid accumulation by inhibiting early adipogenesis in 3T3-L1 cells and zebrafish. Food \& Function 6:2824-2833.

Lee YJ, Kho MC, Tan R, Lee JY, Hwang JinS, Cha JD, Choi KM, Kang DG (2015c) Beneficial effect of combination with Korean Red Ginseng, Gastrodia Rhizoma and Polygoni Multiflori on cholesterol and erectile dysfunction in hyperlipidemia rats. The Korea Journal of Herbology 30:69-75.

Leung PC, Xue CC, Cheng YC (2003) A Comprehensive Guide to Chinese Medicine. 20 ed. World Scientific Publishing Co. Pte. Ltd., Singapore

Li C, Huang Q, Fu X, Yue X-J, Liu RH, You L-J (2015) Characterization, antioxidant and immunomodulatory activities of polysaccharides from Prunella vulgaris Linn. International Journal of Biological Macromolecules 75:298-305

Li C, Lin L-M, Sui F, Wang Z-M, Huo H-R, Dai L, Jiang T-L (2014) Chemistry and pharmacology of Siraitia grosvenorii: A review. Chinese Journal of Natural Medicines 12:89-102

Li CM, Wu JH, Yang RF, Dong XL, He ZY, Tian $X L$, Guo DJ, Wong MS, Qiu TQ, Chan SW (2013a) Ligusticum chuanxiong prevents 
ovariectomy-induced liver and vascular damage in rats. The American Journal of Chinese Medicine 41:831-848

Li D (2006) Research advance on ethnopharmacology, pharmacokinetics and clinical therapeutics of Coix seed and its preparation, Kanglaite injection. Asian Journal of Pharmacodynamics and Pharmacokinetics 6:83-102

Li L, Yan J, Hu K, Gu J, Wang J-J, Deng X-L, Li H, Jing X, Li Z-Y, Ye Q-F, Ouyang D-S (2012) Protective effects of Eucommia lignans against hypertensive renal injury by inhibiting expression of aldose reductase. Journal of Ethnopharmacology 139:454-461

Li S, Li SK, Gan RY, Song FL, Kuang L, Li HB (2013b) Antioxidant capacities and total phenolic contents of infusions from 223 medicinal plants. Industrial Crops and Products 51:289-298

Li W., Zheng H., Bukuru J, De Kimpe N (2004) Natural medicines used in the traditional Chinese medical system for therapy of diabetes mellitus. Journal of Ethnopharmacology 92:1-21

Li X, Wei W (2002) Chinese Materia Medica: Combinations and Applications. Donica Publishing Ltd, St. Albans, UK

Li Y, Xu C, Zhang Q, Liu JY, Tan RX (2005) In vitro anti-Helicobacter pylori action of $\mathbf{3 0}$ Chinese herbal medicines used to treat ulcer diseases. Journal of Ethnopharmacology 98:329-333

Liao C-H, Lin J-Y (2013) Lotus (Nelumbo nucifera Gaertn) plumule polysaccharide ameliorates pancreatic islets loss and serum lipid profiles in non-obese diabetic mice. Food and Chemical Toxicology 58:416-422

Liao J-C, Deng J-S, Chiu C-S, Hou W-C, Huang S-S, Shie P-H, Huang G-J (2012) AntiInflammatory Activities of Cinnamomum cassia Constituents In Vitro and In Vivo. Evidence-Based Complementary and Alternative Medicine 2012:1-12

Lim D, Kim Y (2013) Dried Root of Rehmannia glutinosa Prevents Bone Loss in Ovariectomized Rats. Molecules 18:5804-5813

Lim LS, Shen P, Gong YH, Lee LS, Yong EL (2006) Dynamics of progestogenic activity in serum following administration of Ligusticum chuanxiong. Life Sciences 79:1274-1280
Lim TK (2013) Edible Medicinal and NonMedicinal Plants 7. Flowers. Springer, Dordrecht, Netherlands

Lim TK (2016) Edible Medicinal and NonMedicinal Plants 12. Modified Stems, Roots, Bulbs. Springer, Dordrecht, Netherlands

Lin G-P, Jiang T, Hu X-B, Qiao X-H, Tuo Q-H (2007) Effect of Siraitia Grosvenorii Polysaccharide on Glucose and Lipid of Diabetic Rabbits Induced by Feeding High Fat/High Sucrose Chow. Experimental Diabetes Research 2007:1-4

Lin H-H, Charles AL, Hsieh C-W, Lee Y-C, Ciou J-Y (2015a) Antioxidant effects of 14 Chinese traditional medicinal herbs against human low-density lipoprotein oxidation. Journal of Traditional and Complementary Medicine 5:51-55

Lin H-W, Sun M-X, Wang Y-H, Yang L-M, Yang YR, Huang N, Xuan L-J, Xu Y-M, Bai D-L, Zheng Y-T, Xiao K (2010) Anti-HIV Activities of the Compounds Isolated from Polygonum cuspidatum and Polygonum multiflorum. Planta Medica 76:889-892

Lin L, Harnly JM (2010) Identification of the phenolic components of chrysanthemum flower (Chrysanthemum morifolium Ramat). Food Chemistry 120:319-326

Lin L, Ni B, Lin H, Zhang M, Li X, Yin X, Qu C, Ni J (2015b) Traditional usages, botany, phytochemistry, pharmacology and toxicology of Polygonum multiflorum. A review. Journal of Ethnopharmacology 159:158-183

Lin M, Sun W, Gong W, Zhou Z, Ding Y, Hou Q (2015c) Methylophiopogonanone, a protects against cerebral ischemia/reperfusion injury and attenuates blood-brain barrier disruption in vitro. PLoS One 10:e0124558

Lin R-D, Hou WC, Yen KY, Lee MH (2003) Inhibition of monoamine oxidase B (MAO-B) by Chinese herbal medicines. Phytomedicine 10:650-656

Ling S, Xu J-W (2016) Biological Activities of 2,3,5,4'-Tetrahydroxystilbene-2-O- $\quad \beta \quad$-DGlucoside in Antiaging and Antiaging-Related Disease Treatments. Oxidative Medicine and Cellular Longevity 2016:1-14

Link P, Wetterauer B, Fu Y, Wink M (2015) Extracts of Glycyrrhiza uralensis and Isoliquiritigenin Counteract Amyloid- $\beta$ 
Toxicity in Caenorhabditis elegans. Planta Medica 81:357-362

Liu C, Chen J, Li E, Fan Q, Wang D, Li P, Li X, Chen X, Qiu S, Gao Z, Li H, Hu Y (2015a) The comparison of antioxidative and hepatoprotective activities of Codonopsis pilosula polysaccharide (CP) and sulfated CP. International Immunopharmacology 24:299-305

Liu DD, Ji XW, Li RW (2013a) Effects of Siraitia grosvenorii fruits extracts on physical fatigue in mice. Iranian Journal of Pharmaceutical Research 12:115-121

Liu J-L, Zheng S-L, Fan Q-J, Yuan J-C, Yang SM, Kong F-L (2015b) Optimisation of highpressure ultrasonic-assisted extraction and antioxidant capacity of polysaccharides from the rhizome of Ligusticum chuanxiong. International Journal of Biological Macromolecules 76:80-85

Liu J, Feng L, Zhang M, Ma D, Wang S, Gu J, Fu Q, Qu R, Ma S (2013b) Neuroprotective effect of Liuwei Dihuang decoction on cognition deficits of diabetic encephalopathy in streptozotocin-induced diabetic rat. Journal of Ethnopharmacology 150:371-381

Liu J, Peter K, Shi D, Zhang L, Dong G, Zhang D, Breiteneder H, Jakowitsch J, Ma Y (2014) Traditional Formula, Modern Application: Chinese Medicine Formula Sini Tang Improves Early Ventricular Remodeling and Cardiac Function after Myocardial Infarction in Rats. Evidence-Based Complementary and Alternative Medicine 2014:1-10

Liu Y, Wang Z, Zhang J (Eds.) (2015c) Dietary Chinese Herbs: Chemistry, Pharmacology and Clinical Evidence. Springer, Wien, Austria.

Liu Y, Lv J, Yang B, Liu F, Tian Z, Cai Y, Yang D, Ouyang J, Sun F, Shi Y, Xia P (2015d) Lycium barbarum polysaccharide attenuates type II collagen-induced arthritis in mice. International Journal of Biological Macromolecules 78:318-323

Liu Y-F, Liang D, Luo H, Hao Z-Y, Wang Y, Zhang C-L, Zhang Q-J, Chen R-Y, Yu D-Q (2012) Hepatoprotective Iridoid Glycosides from the Roots of Rehmannia glutinosa. Journal of Natural Products 75:1625-1631

Liu Y, Zhang H-G, Li X-H (2011) A Chinese Herbal Decoction, Danggui Buxue Tang, Improves Chronic Fatigue Syndrome Induced by Food Restriction and Forced Swimming in
Rats. Phytotherapy Research 25:1825-1832

Lu H-J, Tzeng T-F, Liou S-S, Chang CJ, Yang C, Wu M-C, Liu I-M (2014) Ruscogenin Ameliorates Experimental Nonalcoholic Steatohepatitis via Suppressing Lipogenesis and Inflammatory Pathway. BioMed Research International 2014:1-10

Lu H, Zhang L, Huang H (2016) Study on the isolation of active constituents in Lonicera japonica and the mechanism of their antiupper respiratory tract infection action in children. African Health Sciences 15:1295-1301

Lu Y, Zhang B-Y, Jia Z-X, Wu W-J, Lu Z-Q (2011) Hepatocellular carcinoma HepG2 cell apoptosis and caspase-8 and Bcl-2 expression induced by injectable seed extract of Coix lacryma-jobi. Hepatobiliary \& Pancreatic Diseases International 10:303-307

Luo H, Lin S, Ren F, Wu L, Chen L, Sun Y (2007) Antioxidant and Antimicrobial Capacity of Chinese Medicinal Herb Extracts in Raw Sheep Meat. Journal of Food Protection 70:1440-1445

Luszczki JJ, Wojda E, Andres-Mach M, Cisowski W, Glensk M, Glowniak K, Czuczwar SJ (2009) Anticonvulsant and acute neurotoxic effects of imperatorin, osthole and valproate in the maximal electroshock seizure and chimney tests in mice: A comparative study. Epilepsy Research 85:293-299

Lv L, Cheng Y, Zheng T, Li X, Zhai R (2014) Purification, antioxidant activity and antiglycation of polysaccharides from Polygonum multiflorum Thunb. Carbohydrate Polymers 99:765-773

Ma X, Zhang K, Li H, Han S, Ma Z, Tu P (2013) Extracts from Astragalus membranaceus limit myocardial cell death and improve cardiac function in a rat model of myocardial ischemia. Journal of Ethnopharmacology 149:720-728

Maffi L (2001) On biocultural diversity. Linking language, knowledge, and the environment. Smithsonian Inst Press, Washington DC, USA

Maffi L (2005) Linguistic, cultural, and biological diversity. Annual Review of Anthropology 34:599-617

Mahajan R, Gajare S (2012) Manifestation of erectile dysfunction with adaptogenic antioxidant aphrodisiac plants. International Journal of Pharmaceutical and Biomedical 


\section{Research 3:52-68}

Mahajan RT, Chopda MZ (2009) PhytoPharmacology of Ziziphus jujuba Mill. A plant review. Pharmacognosy Review 3:320-329

Mahboob T, Azlan A-M, Tan T-C, Samudi C, Sekaran SD, Nissapatorn V, Wiart C (2016) Antiencystment and amoebicidal activity of Lonicera japonica Thunb. and its major constituent chlorogenic acid in vitro. Asian Pacific Journal of Tropical Medicine 9:866-871

Manosroi A, Sainakham M, Chankhampan C, Abe M, Manosroi W, Manosroi J (2016) Potent in vitro anti-proliferative, apoptotic and antioxidative activities of semi-purified Job's tears (Coix lachryma-jobi Linn.) extracts from different preparation methods on 5 human cancer cell lines. Journal of Ethnopharmacology 187:281-292

Mao F, Xiao B, Jiang Z, Zhao J, Huang X, Guo J (2011) Anticancer effect of Lycium barbarum polysaccharides on colon cancer cells involves G0/G1 phase arrest. Medical Oncology 28:121-126

Marcos M, Mera G (2015) Migrantes internacionales en la Aglomeración Gran Buenos Aires. Cuadernos Geográficos de la Universidad de Granada 54:257-282

Marzouk TMF, El-Nemer AMR, Baraka HN (2013) The Effect of Aromatherapy Abdominal Massage on Alleviating Menstrual Pain in Nursing Students: A Prospective Randomized Cross-Over Study. Evidence-Based Complementary and Alternative Medicine 2013:1-6

Maslov LN, Lishmanov YB, Arbuzov AG, Krylatov A V., Budankova E V., Konkovskaya YN, Burkova VN, Severova EA (2009) Antiarrhythmic Activity of Phytoadaptogens in Short-Term Ischemia-Reperfusion of the Heart and Postinfarction Cardiosclerosis. Bulletin of Experimental Biology and Medicine 147:331-334

Matsuura H, Saxena G, Farmer S, Hancock R, Towers G (1996) Antibacterial and Antifungal Polyine Compounds from Glehnia littoralis ssp. leiocarpa. Planta Medica 62:256-259

Mayo JL (1999) A natural approach to menopause. Applied Nutritional Science Reports $5: 1-8$

Mazaro-Costa R, Andersen ML, Hachul H, Tufik $S$ (2010) Medicinal Plants as Alternative Treatments for Female Sexual Dysfunction:
Utopian Vision or Possible Treatment in Climacteric Women? The Journal of Sexual Medicine 7:3695-3714

McKenna DJ, Hughes K, Jones K (2002) Astragalus. Alternative Therapies in Health and Medicine 8:34-40

Meng G, Wang M, Zhang K, Guo Z, Shi J (2014) Research Progress on the Chemistry and Pharmacology of Prunella vulgaris Species. Open Access Library 1:1-19

Mimaki Y, Sashida Y, Kuroda M, Nishino A, Satomi Y, Nishino H (1995) Inhibitory Effects of Steroidal Saponins on 12-OTetradecanoylphorbol-13-acetate (TPA)Enhanced 32P-Incorporation into Phospholipids of HeLa Cells and Proliferation of Human Malignant Tumor Cells. Biological \& Pharmaceutical Bulletin 18:467-469

Mimaki Y, Yokosuka A, Kuroda M, Sashida Y (2001) Cytotoxic Activities and StructureCytotoxic Relationships of Steroidal Saponins. Biological \& Pharmaceutical Bulletin 24:1286-1289

Miyazawa M, Hisama M (2003) Antimutagenic Activity of Flavonoids from Chrysanthemum morifolium. Bioscience, Biotechnology, and Biochemistry 67:2091-2099

Moon H-I (2010) Three diterpenes from Leonurus japonicus houtt protect primary cultured rat cortical cells from glutamateinduced toxicity. Phytotherapy Research 24:1256-1259

Moon S-S, Lee J-Y, Cho S-C (2004) Isotsaokoin, an Antifungal Agent from Amomum tsao-ko. Journal of Natural Products 67:889-891

Mutreja A, Agarwal M, Kushwaha S, Chauhan A (2008) Effect of Nelumbo nucifera seeds on the reproductive organs of female rats. Iranian Journal of Reproductive Medicine 6:7-11

Naftali T, Feingelernt H, Lesin Y, Rauchwarger A, Konikoff FM (2008) Ziziphus jujuba Extract for the Treatment of Chronic Idiopathic Constipation: A Controlled Clinical Trial. Digestion 78:224-228

Nagai T, Suzuki N, Kai N, Tanoue Y (2014) Functional properties of autolysate and enzymatic hydrolysates from yam tsukuneimo (Dioscorea opposita Thunb.) tuber mucilage tororo: antioxidative activity and antihypertensive activity. Journal of Food 


\section{Science and Technology 51:3838-3845}

Nantia EA, Moundipa PF, Monsees TK, Carreau $S$ (2009) Medicinal plants as potential male anti-infertility agents: a review. Basic and Clinical Andrology 19:148-158

Nardi GM, G F-JA, Freire CG, Megiolaro F, Schneider K, Perazzoli MR, Nascimento SR, Gon AC, Mariano LN, Wagner G, Niero R, Locatelli C (2016) Anti-inflammatory activity of berry fruits in mice model of inflammation is based on oxidative stress modulation. Pharmacognosy Research 8:42-49

$\mathrm{Ng} \mathrm{T}$., Liu F, Wang H. (2004) The antioxidant effects of aqueous and organic extracts of Panax quinquefolium, Panax notoginseng, Codonopsis pilosula, Pseudostellaria heterophylla and Glehnia littoralis. Journal of Ethnopharmacology 93:285-288

Ngoc TM, Nhiem NX, Khoi NM, Son DC, Hung T $V$, Van-Kiem P (2014) A new coumarin and cytotoxic activities of constituents from Cinnamomum cassia. Natural Product Communications 9:487-488

Palve A, Shetty P, Pimpliskar M, Jadhav RN (2015) Study on antibacterial and antifungal activities of Sterculia lychnophora extracts. International Journal of Current Microbiology and Applied Sciences 4:336-341

Panossian A (2003) Adaptogens: Tonic Herbs for Fatigue and Stress. Alternative and Complementary Therapies 9:327-331

Panossian A (2013) Adaptogens in Mental and Behavioral Disorders. Psychiatric Clinics of North America 36:49-64

Park H-J, Zhang N, Park DK (2011) Topical application of Polygonum multiflorum extract induces hair growth of resting hair follicles through upregulating Shh and $\beta$-catenin expression in C57BL/6 mice. Journal of Ethnopharmacology 135:369-375

Park J-W, Bae H, Lee G, Hong B-G, Yoo HH, Lim S-J, Lee K, Kim J, Ryu B, Lee B-J, Bae J, Lee H, $\mathrm{Bu} Y$ (2013) Prophylactic effects of Lonicera japonica extract on dextran sulphate sodiuminduced colitis in a mouse model by the inhibition of the Th1/Th17 response. British Journal of Nutrition 109:283-292

Park SJ, Ahn YJ, Lee HE, Hong E, Ryu JH (2015) Standardized Prunella vulgaris var. lilacina Extract Enhances Cognitive Performance in Normal Naive Mice.

\section{Phytotherapy Research 29:1814-1821}

Park SJ, Kim DH, Lee IK, Jung WY, Park DH, Kim JM, Lee KR, Lee K-T, Shin CY, Cheong JH, Ko KH, Ryu JH (2010) The ameliorating effect of the extract of the flower of Prunella vulgaris var. lilacina on drug-induced memory impairments in mice. Food and Chemical Toxicology 48:1671-1676

Parker S, May B, Zhang C, Zhang AL, Lu C, Xue CC (2016) A Pharmacological Review of Bioactive Constituents of Paeonia lactiflora Pallas and Paeonia veitchii Lynch. Phytotherapy Research 30:1445-1473

Patel D, Kumar R, Prasad S, Hemalatha S (2011) Pharmacologically screened aphrodisiac plant - A review of current scientific literature. Asian Pacific Journal of Tropical Biomedicine 1:131-138

Patwardhan B, Warude D, Pushpangadan D, Bhatt N (2005) Ayurveda and Traditional Chinese Medicine: A Comparative Overview. Evidence-Based Complementary and Alternative Medicine 2:465-473

Paudel KR, Panth N (2015) Phytochemical Profile and Biological Activity of Nelumbo nucifera. Evidence-Based Complementary and Alternative Medicine 2015:1-16

Paulpriya K, Mohan VR (2012) In vitro antioxidante potential of metanol extract of Dioscorea oppositifolia. Science Research Reporter 2:239-245

Peng Z, Shen H, Gu J (2015) Clinical observation of breast hyperplasia treated with auricular point sticking therapy and Xiaopijian. Zhongguo Zhen jiu 35:778-780

Pérez-Hernández J, Zaldívar-Machorro VJ, Villanueva-Porras D, Vega-Ávila E, Chavarría A (2016) A Potential Alternative against Neurodegenerative Diseases: Phytodrugs. Oxidative Medicine and Cellular Longevity 2016:1-19

Peter K, Schinnerl J, Felsinger S, Brecker L, Bauer R, Breiteneder H, Xu R, Ma Y (2013) A novel concept for detoxification: Complexation between aconitine and liquiritin in a Chinese herbal formula ("Sini Tang"). Journal of Ethnopharmacology 149:562-569

Pochettino ML, Puentes JP, Buet Costantino F, Arenas PM, Ulibarri EA, Hurrell JA (2012) Functional Foods and Nutraceuticals in a Market of Bolivian Immigrants in Buenos 
Aires (Argentina). Evidence-Based Complementary and Alternative Medicine 2012:1-14

Pole S (2012) Ayurvedic Medicine. The Principles of Traditional Practice. Singing Dragon, London, UK

Poornima P, Kumar JD, Zhao Q, Blunder M, Efferth T (2016) Network pharmacology of cancer: From understanding of complex interactomes to the design of multi-target specific therapeutics from nature. Pharmacological Research 111:290-302

Preetha TS, Hemanthakumar AS, Krishnan PN (2013) A comprehensive review of Kaempferia galanga L. (Zingiberaceae): A high sought medicinal plant in Tropical Asia. Journal of Medicinal Plants 4:270-276

Provino R (2010) The role of adaptogens in stress management. Australian Journal of Medical Herbalism 22:41-49

PubMed (2016) US National Library of Medicine National Institutes of Health. NCBI, National Center for Biotechnology Information.

[https://www.ncbi.nlm.nih.gov/pubmed] Accessed July 1, 2016

Puentes JP, Hurrell JA (2015) Plantas andinas y sus productos comercializados con fines medicinales $y$ alimentarios en el Área Metropolitana Buenos Aires-La Plata, Argentina. Boletín Latinoamericano y del Caribe de Plantas Medicinales y Aromáticas 14:206-236

Qian L, Yu S (2016) Protective effect of polysaccharides from Lycium barbarum on spermatogenesis of mice with impaired reproduction system induced by cyclophosphamide. American Journal of Reproductive Immunology 76:383-385

Qu H-M, Liu S-J, Zhang C-Y (2014) Antitumor and antiangiogenic activity of Schisandra chinensis polysaccharide in a renal cell carcinoma model. International Journal of Biological Macromolecules 66:52-56

Quinlan M (2005) Considerations for collecting freelists in the field: examples from Ethnobotany. Field Methods 17:1-16

Rahman S, Begum H, Rahman Z, Ara F, Iqbal MJ, Yousuf AKM (2013) Effect of Cinnamon (Cinnamomum cassia) as a Lipid Lowering Agent on Hypercholesterolemic Rats. Journal of Enam Medical College 3:94-98
Rajput MS, Sinha S, Mathur V, Agrawal P (2011) Herbal Antidepressants. International Journal of Pharmaceutical Frontier Research 1:159-169

Ran X, Ma L, Peng C, Zhang H, Qin L-P (2011) Ligusticum chuanxiong Hort: A review of chemistry and pharmacology. Pharmaceutical Biology 49:1180-1189

Rauwald HW, Savtschenko A, Merten A, Rusch C, Appel K, Kuchta K (2015) GABA(a) Receptor Binding Assays of Standardized Leonurus cardiaca and Leonurus japonicus Extracts as Well as Their Isolated Constituents. Planta Medica 81:1103-1110

Ryu KH, Rhee HI, Kim JH, Yoo H, Lee BY, Um KA, Kim K, Noh JY, Lim KM, Chung JH (2010) Anti-inflammatory and analgesic activities of SKLJI, a highly purified and injectable herbal extract of Lonicera japonica. Bioscience, Biotechnology, and Biochemistry 74:2022-2028

Sabzghabaee A, Khayam I, Kelishadi R, Ghannadi A, Soltani R, Badri S, Shirani S (2013) Effect of Zizyphus Jujuba Fruits on Dyslipidemia in Obese Adolescents: a Triplemasked Randomized Controlled Clinical Trial. Medical Archives 67:156-159

Sakamoto S, Kudo $H$, Suzuki S, Sassa $S$, Yoshimura S, Nakayama T, Maemura M, Mitamura T, Qi Z, Liu X, Yagishita Y, Asai A (1996) Pharmacotherapeutic Effects of Tokishakuyaku-san on Leukorrhagia in Young Women. The American Journal of Chinese Medicine 24:165-168

Santos-Neto LL dos, Vilhena-Toledo MA, Medeiros-Souza P, Souza GA (2006) The Use of Herbal Medicine in Alzheimer's Disease - A Systematic Review. Evidence-Based Complementary and Alternative Medicine 3:441-445

Sassone S, Mera C (2007) Barrios de migrantes en Buenos Aires. Identidad, cultura, cohesión socio-territorial. V Congreso Europeo CEISAL de Latinoamericanistas, Bruxelles, Belgique

Schröder S, Beckmann K, Franconi G, MeyerHamme G, Friedemann T, Greten HJ, Rostock M, Efferth T (2013) Can Medical Herbs Stimulate Regeneration or Neuroprotection and Treat Neuropathic Pain in Chemotherapy-Induced Peripheral Neuropathy? Evidence-Based Complementary and Alternative Medicine 2013:1-18 
Semwal A, Kumar R, Singh R (2013) Nature's Aphrodisiacs. A review of current scientific literature. Int J Recent Adv Pharm Res 3:1-20

Shang H, Cao S, Wang J, Zheng H, Putheti R (2010) Glabridin from Chinese herb licorice inhibits fatigue in mice. African Journal of Traditional, Complementary and Alternative Medicines 7:17-23

Shang X, Pan H, Li M, Miao X, Ding H (2011) Lonicera japonica Thunb.: Ethnopharmacology, phytochemistry and pharmacology of an important traditional Chinese medicine. Journal of Ethnopharmacology 138:1-21

Shang X, Pan H, Wang X, He H, Li M (2014) Leonurus japonicus Houtt.: Ethnopharmacology, phytochemistry and pharmacology of an important traditional Chinese medicine. Journal of Ethnopharmacology 152:14-32

Sharma B, Gupta S, Sharma K (2012) Natural folk remedy for menorrhagia. Journal of Information, Knowledge and Research in Humanities and Social Sciences 2:86-88

Shergis JL, Liu S, Chen X, Zhang AL, Guo X, Lu C, Xue CC (2015) Dang Shen [Codonopsis pilosula (Franch.) Nannf] Herbal Formulae for Chronic Obstructive Pulmonary Disease: A Systematic Review and Meta-analysis. Phytotherapy Research 29:167-186

Shetty P, Palve A, Pimpliskar M, Jadhav RN, Shinde $P$ (2014) In-silico docking analysis of Sterculia lychnophora compounds against proteins causing Alzheimer's disease. International Journal of Engineering Science and Innovative Technology 3:58-164

Shih C-H, Lin Y-J, Chen C-M, Ko W-C (2015) Butylidenephthalide antagonizes cromakaliminduced systolic pressure reduction in conscious normotensive rats. BMC Complementary and Alternative Medicine 15:344

Shin IS, Lee MY, Ha HK, Seo CS, Shin H-K (2012) Inhibitory effect of Yukmijihwang-tang, a traditional herbal formula against testosterone-induced benign prostatic hyperplasia in rats. BMC Complementary and Alternative Medicine 12:1068

Shin Y, Son H, Kim J, Heo J, Lee S, Kim J (2015) Cinnamomum cassia bark produced by solidstate fermentation with Phellinus baumii has the potential to alleviate atopic dermatitis- related symptoms. International Journal of Molecular Medicine 35:187-194

Singh B, Gupta V, Bansal P, Singh S, Kumar D (2010) Pharmacological potential of plants used as aphrodisiacs. International Journal of Pharmaceutical Sciences Review and Research 5:104-113

Singh CB, Chanu SB, Bidyababy T, Radhapiyari DW, Brojendro SS, Nongalleima K, Lokendrajit N, Swapana N, Singh LW (2013a) Biological and Chemical properties of Kaempferia galanga L. a Zingiberaceae plant. NeBIO 4:35-41

Singh S, Ali A, Singh R, Kaur R (2013b) Sexual abnormalities in males and their herbal therapeutic aspects. Pharmacologia 4:165-275

Sirisha-Chowdary

G

(2013)

Neuropharmacological screening of ethanolic extract of Nelumbo nucifera Gaertner seeds. Indian Journal of Research in Pharmacy and Biotechnology 1:635-642

Sohn D-W, Kim H-Y, Kim S-D, Lee E-J, Kim H-S, Kim J-K, Hwang S-Y, Cho Y-H, Kim S-W (2008) Elevation of intracavernous pressure and NOcGMP activity by a new herbal formula in penile tissues of spontaneous hypertensive male rats. Journal of Ethnopharmacology 120:176-180

Song F, Li H, Sun J, Wang S (2013) Protective effects of cinnamic acid and cinnamic aldehyde on isoproterenol-induced acute myocardial ischemia in rats. Journal of Ethnopharmacology 150:125-130

Sreekeesoon DP, Mahomoodally MF (2014) Ethnopharmacological analysis of medicinal plants and animals used in the treatment and management of pain in Mauritius. Journal of Ethnopharmacology 157:181-200

Stepp JR (2005) Advances in ethnobiological field methods. Field Methods 17:211-218

Su X, Li X, Tao H, Zhou J, Wu T, Chou G, Cheng $Z$ (2013) Simultaneous isolation of seven compounds from Glehnia littoralis roots by off-line overpressured layer chromatography guided by a TLC antioxidant autographic assay. Journal of Separation Science 36:3644-3650

Sugimoto Y, Furutani S, Nishimura K, Itoh A, Tanahashi T, Nakajima $H$, Oshiro $H$, Sun $S$, Yamada J (2010) Antidepressant-like effects of neferine in the forced swimming test involve the serotonin1A (5-HT1A) receptor in mice. 
European Journal of Pharmacology 634:62-67

Sun BS, Chen YP, Wang YB, Tang SW, Pan FY, Li Z, Sung CK (2012) Anti-obesity effects of mogrosides extracted from the fruits of Siraitia grosvenorii (Cucurbitaceae). African Journal of Pharmacy and Pharmacology 6:1492-1501

Sun H, Luo G, Chen D, Xiang Z (2016) A Comprehensive and System Review for the Pharmacological Mechanism of Action of Rhein, an Active Anthraquinone Ingredient. Frontiers in Pharmacology 7:247

Sun K, Cao S, Pei L, Matsuura A, Xiang L, Qi J (2013) A Steroidal Saponin from Ophiopogon japonicus Extends the Lifespan of Yeast via the Pathway Involved in SOD and UTH1. International Journal of Molecular Sciences 14:4461-4475

Sun QL, Hua $S$, Ye JH, Zheng XQ, Liang YR (2010) Flavonoids and volatiles in Chrysanthemum morifolium Ramat. flower from Tongxiang County in China. African Journal of Biotechnology 9:3817-3821

Sun X, Gao R-L, Xiong Y-K, Huang Q-C, Xu M (2014) Antitumor and immunomodulatory effects of a water-soluble polysaccharide from Lilii Bulbus in mice. Carbohydrate Polymers 102:543-549

Sun Y, Zhang J, Huo R, Zhai T, Li H, Wu P, Zhu X, Zhou Z, Shen B, Li N (2015) Paeoniflorin inhibits skin lesions in imiquimod-induced psoriasis-like mice by downregulating inflammation. International Immunopharmacology 24:392-399

Sung Y-Y, Yoon T, Jang JY, Park S-J, Kim HK (2011) Topical application of Rehmannia glutinosa extract inhibits mite allergeninduced atopic dermatitis in $\mathrm{NC} / \mathrm{Nga}$ mice. Journal of Ethnopharmacology 134:37-44

Taejarernwiriyakul O, Anzai $\mathrm{N}$, Jutabha $P$, Kruanamkam W, Chanluang S (2015) Hypouricemia and nephroprotection of Coix lacryma-jobi L. seed extract. Songklanakarin J. Sci. Technol. 37:441-447

Tahergorabi Z, Abedini MR, Mitra M, Fard MH, Beydokhti H (2015) "Ziziphus jujuba": A red fruit with promising anticancer activities. Pharmacognosy Reviews 9:99-106

Takasaki M, Konoshima T, Murata Y, Sugiura M, Nishino H, Tokuda H, Matsumoto K, Kasai R, Yamasaki K (2003) Anticarcinogenic activity of natural sweeteners, cucurbitane glycosides, from Momordica grosvenori. Cancer Letters 198:37-42

Takeo E, Yoshida H, Tada N, Shingu T, Matsuura $\mathrm{H}$, Murata $\mathrm{Y}$, Yoshikawa S, Ishikawa T, Nakamura H, Ohsuzu F, Kohda H (2002) Sweet Elements of Siraitia Grosvenori Inhibit Oxidative Modification of Low-Density Lipoprotein. Journal of Atherosclerosis and Thrombosis 9:114-120

Tan L, Chen W, Wei M-Y, Shen J, Yu M-F, Yang G, Guo D, Qin G, Ji G, Liu Q-H (2015) Relaxant Action of Plumula nelumbinis Extract on Mouse Airway Smooth Muscle. EvidenceBased Complementary and Alternative Medicine 2015:1-10

Tang W, Eisenbrand G (2013) Chinese Drugs of Plant Origin: Chemistry, Pharmacology, and Use in Traditional and Modern Medicine. Springer, Berlin, Germany

Terashima S, Shimizu M, Horie S, Morita M (1991) Terashima S, Shimizu M, Horie S, Morita M (1991) Studies on aldose-reductase inhibitors from natural products. IV. Constituents and aldose-reductase inhibitory effect of Chrysanthemum morifolium, Bixa orellana and Ipomoea batatas. Chemical \& Pharmaceutical Bulletin 39:3346-3347

The Plant List (2013) The Plant List. Version 1.1. [http://www.theplantlist.org] Accessed July 1 , 2016

Thiengsusuk A, Chaijaroenkul W, Na-Bangchang K (2013) Antimalarial activities of medicinal plants and herbal formulations used in Thai traditional medicine. Parasitology Research 112:1475-1481

Tho N, An T, Tri M, Sreekanth T, Lee J, Nagajyothi P, Lee K (2013) Green synthesis of silver nanoparticles using Nelumbo nucifera seed extract and its antibacterial activity. Acta Chimica Slovenica 60:673-678

Tian J, Che H, Ha D, Wei Y, Zheng S (2012) Characterization and anti-allergic effect of a polysaccharide from the flower buds of Lonicera japonica. Carbohydrate Polymers 90:1642-1647

Tu X, Huang G, Tan S (2009) Chinese Herbal Medicine for Dysfunctional Uterine Bleeding: A Meta-Analysis. Evidence-Based Complementary and Alternative Medicine 6:99-105 
Tzeng TF, Liou SS, Chang CJ, Liu IM (2014) The ethanol extract of Lonicera japonica (Japanese honeysuckle) attenuates diabetic nephropathy by inhibiting p-38 MAPK activity in streptozotocin-induced diabetic rats. Planta Medica 80:121-129

Um YR, Kong C-S, Lee JI, Kim YA, Nam TJ, Seo $Y$ (2010) Evaluation of chemical constituents from Glehnia littoralis for antiproliferative activity against HT-29 human colon cancer cells. Process Biochemistry 45:114-119

Umar MI, Asmawi MZB, Sadikun A, Altaf R, Iqbal MA (2011) Phytochemistry and medicinal properties of Kaempferia galanga $L$. (Zingiberaceae) extracts. African Journal of Pharmacy and Pharmacology 5:1638-1647

Vijaya, Yadav AK (2016) In vitro anthelmintic assessment of selected phytochemicals against Hymenolepis diminuta, a zoonotic tapeworm. Journal of Parasitic Diseases 40:1082-1086

Wang D, Du Q, Li H, Wang S (2016a) The Isosteroid Alkaloid Imperialine from Bulbs of Fritillaria cirrhosa Mitigates Pulmonary Functional and Structural Impairment and Suppresses Inflammatory Response in a COPD-Like Rat Model. Mediators of Inflammation 2016:1-17

Wang D, Wang S, Feng Y, Zhang L, Li Z, Ma J, Luo Y, Xiao W (2014a) Antitumor effects of Bulbus Fritillariae cirrhosae on Lewis lung carcinoma cells in vitro and in vivo. Industrial Crops \& Products 54:92-101

Wang D, Yang J, Du Q, Li H, Wang S (2016b) The total alkaloid fraction of bulbs of Fritillaria cirrhosa displays anti-inflammatory activity and attenuates acute lung injury. Journal of Ethnopharmacology 193:150-158

Wang D, Zhu J, Wang S, Wang X, Ou Y, Wei D, Li $X$ (2011) Antitussive, expectorant and antiinflammatory alkaloids from Bulbus Fritillariae Cirrhosae. Fitoterapia 82:1290-1294

Wang JM, Zhu XR (2012) Antidepression activity of extract of Heshouwu (Radix Polygoni Multiflori). Journal of Beijing University of Traditional Chinese Medicine 7:449-451

Wang M, Xing S, Luu T, Fan M, Li X (2015a) The gastro-intestinal tract metabolism and pharmacological activities of grosvenorine, a major and characteristic flavonoid in the fruits of Siraitia grosvenorii. Chemistry \& Biodiversity 12:1652-1664.

Wang Q, Shen L, Ma S, Chen M, Lin X, Hong Y, Liang S, Feng $Y(2015 b)$ Effects of Ligusticum chuanxiong and Gastrodia elata on bloodbrain barrier permeability in migraine rats. Pharmazie 70:421-426

Wang R-F, Wu X-W, Geng D (2013) Two Cerebrosides Isolated from the Seeds of Sterculia lychnophora and Their Neuroprotective Effect. Molecules 18:1181-1187

Wang TT, Huang HH, Zhang Y, Li X, Li H, Jiang Q, Gao WY (2015c) Role of effective composition on antioxidant, antiinflammatory, sedative-hypnotic capacities of 6 common edible Lilium varieties. Journal of Food Science 80:H857-H868.

Wang Y, Shi L-L, Wang L-Y, Xu J-W, Feng Y (2015d) Protective Effects of MDG-1, a Polysaccharide from Ophiopogon japonicus on Diabetic Nephropathy in Diabetic KKAy Mice. International Journal of Molecular Sciences 16:22473-22484

Wang Y, Zhang R, Xie J, Lu J, Yue Z (2014b) Analgesic activity of catalpol in rodent models of neuropathic pain, and its spinal mechanism. Cell Biochemistry and Biophysics 70:1565-1571

Wang Y, Zhu Y, Ruan K, Wei H, Feng Y (2014c) MDG-1, a polysaccharide from Ophiopogon japonicus, prevents high fat diet-induced obesity and increases energy expenditure in mice. Carbohydrate Polymers 114:183-189

Wang Z-T, Du Q, Xu G-J, Wang R-J, Fu D-Z, Ng T-B (1997) Investigations on the protective action of Condonopsis pilosula (Dangshen) extract on experimentally-induced gastric ulcer in rats. General Pharmacology: The Vascular System 28:469-473

Wardlaw AJ, Dunnette S, Gleich GJ, Collins J V., Kay $A B(1988)$ Eosinophils and Mast Cells in Bronchoalveolar Lavage in Subjects with Mild Asthma: Relationship to Bronchial Hyperreactivity. American Review of Respiratory Disease 137:62-69

Washida K, Itoh Y, Iwashita T, Nomoto K (2009) Androgen modulators from the roots of Paeonia lactiflora (Paeoniae Radix) grown and processed in Nara Prefecture. Chemical and Pharmaceutical Bulletin (Japan) 57:971-974 
Wei Y, Liu J, Zhang H, Du X, Luo Q, Sun J, Liu F, Li M, Xu F, Wei K, Dong J (2016) Ligustrazine attenuates inflammation and the associated chemokines and receptors in ovalbumineinduced mouse asthma model. Environmental Toxicology and Pharmacology 46:55-61

Weon JB, Yang HJ, Lee B, Yun BR, Ahn JH, Lee HY, Ma CJ (2011) Neuroprotective activity of the methanolic extract of Lonicera japonica in glutamate-injured primary rat cortical cells. Pharmacognosy Magazine 7:284-288

Wojcikowski K, Wohlmuth $\mathrm{H}$, Johnson DW, Rolfe M, Gobe G (2009) An in vitro investigation of herbs traditionally used for kidney and urinary system disorders: Potential therapeutic and toxic effects. Nephrology 14:70-79

Wong C-C, Li H-B, Cheng K-W, Chen F (2006) A systematic survey of antioxidant activity of 30 Chinese medicinal plants using the ferric reducing antioxidant power assay. Food Chemistry 97:705-711

Wong KL, Lai YM, Li KW, Fai Lee K, Ng TB, Pan Cheung $\mathrm{H}$, Bo Zhang $\mathrm{Y}$, Lao L, Ngok-Shun Wong R, Chui Shaw P, Ho Wong J, Zhang Z-J, Lam JKW, Wencai Y, Wing Sze SC (2015) A Novel, Stable, Estradiol-Stimulating, Osteogenic Yam Protein with Potential for the Treatment of Menopausal Syndrome. Scientific Reports 5:10179

Wong M (1976) La médecine chinoise par les plantes. Éditions Tchou, Paris, France

Wong VKC, Yu L, Cho CH (2008) Protective effect of polysaccharides from Angelica sinensis on ulcerative colitis in rats. Inflammopharmacology 16:162-167

Wu JN (2005) An Illustrated Chinese Materia Medica. Oxford University Press, New York, USA

Wu Y-C, Hsieh C-L (2011) Pharmacological effects of Radix Angelica sinensis (Danggui) on cerebral infarction. Chinese Medicine 6:32

Wu Y, Cui SW, Tang J, Wang Q, Gu X (2007) Preparation, partial characterization and bioactivity of water-soluble polysaccharides from boat-fruited sterculia seeds. Carbohydrate Polymers 70:437-443

Wu ZY, Raven PH, Hong DY (1995-2013) Flora of China. Vol. 2-25. [http://www.efloras.org/flora_page.aspx?flora_id= 2] Accessed July 20, 2016
Xiao H-J, Wang T, Chen J, Fan L-C, Yin C-P, Liu $\mathrm{J}-\mathrm{H}$, Gao X (2010) Chuanxiongzine relaxes isolated corpus cavernosum strips and raises intracavernous pressure in rabbits. International Journal of Impotence Research 22:120-126

Xiao J, Liong EC, Ching YP, Chang RCC, So KF, Fung ML, Tipoe GL (2012) Lycium barbarum polysaccharides protect mice liver from carbon tetrachloride-induced oxidative stress and necroinflammation. Journal of Ethnopharmacology 139:462-470

Xie G, Jiang N, Wang S, Qi R, Wang L, Zhao P, Liang L, Yu B (2015) Eucommia ulmoides Oliv. bark aqueous extract inhibits osteoarthritis in a rat model of osteoarthritis. Journal of Ethnopharmacology 162:148-154

Xin T, Zhang F, Jiang Q, Chen C, Huang D, Li Y, Shen W, Jin Y, Sui G (2012) The inhibitory effect of a polysaccharide from Codonopsis pilosula on tumor growth and metastasis in vitro. International Journal of Biological Macromolecules 51:788-793

Xu L, Grandi N, Del Vecchio C, Mandas D, Corona A, Piano D, Esposito F, Parolin C, Tramontano E (2015) From the traditional Chinese medicine plant Schisandra chinensis new scaffolds effective on HIV-1 reverse transcriptase resistant to non-nucleoside inhibitors. Journal of Microbiology 53:288-293

Xutian S, Tai S, Yuan CS (2014) Handbook of Traditional Chinese Medicine. Vol. 1. World Scientific, Singapore

Yamamoto J, Tadaishi M, Yamane T, Oishi Y, Shimizu M, Kobayashi-Hattori K (2015) Hot water extracts of edible Chrysanthemum morifolium Ramat. exert antidiabetic effects in obese diabetic KK-A y mice. Bioscience, Biotechnology, and Biochemistry 79:1147-1154

Yan Y-M, Fang P, Yang M-T, Li N, Lu Q, Cheng Y$X \quad$ (2015) Anti-diabetic nephropathy compounds from Cinnamomum cassia. Journal of Ethnopharmacology 165:141-147

Yang C-H, Li R-X, Chuang L-Y (2012a) Antioxidant Activity of Various Parts of Cinnamomum cassia Extracted with Different Extraction Methods. Molecules 17:7294-7304

Yang C, Gou Y, Chen J, An J, Chen W, Hu F (2013a) Structural characterization and antitumor activity of a pectic polysaccharide from Codonopsis pilosula. Carbohydrate 


\section{Polymers 98:886-895}

Yang F, Ding S, Liu W, Liu J, Zhang W, Zhao Q, Ma X (2015a) Antifungal activity of 40 TCMs used individually and in combination for treatment of superficial fungal infections. Journal of Ethnopharmacology 163:88-93

Yang $\mathrm{H}$, Zhou QM, Peng C, Liu LS, Xie XF, Xiong L, Liu ZH (2014) Coumarins from Leonurus japonicus and their anti-platelet aggregative activity. Zhongguo Zhong yao za zhi 39:4356-4359

Yang H-J, Kim M-J, Kwon D-Y, Kim da S, Lee YH, Kim JE, Park S (2016a) Anti-diabetic activities of Gastrodia elata Blume water extracts are mediated mainly by potentiating glucose-stimulated insulin secretion and increasing $\beta$-cell mass in non-obese Type 2 Diabetic animals. Nutrients 8:161

Yang J, Huang H, Zhu LJ (2013b) Introduction to Chinese Materia Medica. World Scientific, Singapore.

Yang Q-H, Liang Y, Xu Q, Zhang Y, Xiao L, Si L-Y (2011) Protective effect of tetramethylpyrazine isolated from Ligusticum chuanxiong on nephropathy in rats with streptozotocininduced diabetes. Phytomedicine 18:1148-1152

Yang T, Jia M, Zhou S, Pan F, Mei Q (2012b) Antivirus and immune enhancement activities of sulfated polysaccharide from Angelica sinensis. International Journal of Biological Macromolecules 50:768-772

Yang UJ, Maeng H, Park TS, Shim SM (2015b) Houttuynia cordata extract improves physical endurance performance by regulating endothelial production of nitric oxide. Journal of Medicinal Food 18:1022-1031

Yang X (2013) Encyclopedic Reference of Traditional Chinese Medicine. Springer, Berlin, Germany

Yang $X$, Küenzi $P$, Plitzko I, Potterat $O$, Hamburger M (2009) Bicyclononane aldehydes and antiproliferative constituents from Amomum tsao-ko. Planta Medica 75:543-546

Yang Y, Yan R-W, Cai X-Q, Zheng Z-L, Zou G-L (2008) Chemical composition and antimicrobial activity of the essential oil of Amomum tsao-ko. Journal of the Science of Food and Agriculture 88:2111-2116

Yang Y, Yue Y, Runwei Y, Guolin Z (2010) Cytotoxic, apoptotic and antioxidant activity of the essential oil of Amomum tsao-ko. Bioresource Technology 101:4205-4211

Yang Y, Park BI, Hwang EH, You YO (2016b) Composition analysis and inhibitory effect of Sterculia lychnophora against biofilm formation by Streptococcus mutans. Evidence-Based Complementary and Alternative Medicine 2016:1-9

Yarnell E, Abascal K (2005) Natural Approaches to Treating Chronic Prostatitis and Chronic Pelvic Pain Syndromes. Alternative and Complementary Therapies 11:246-251

Yarnell E, Abascal K (2013) Chinese Herbal Formulas Every Western Practitioner Should Know-Part 1. Alternative and Complementary Therapies 19:194-201

Ye S, Mao B, Yang L, Fu W, Hou J (2016) Thrombosis recanalization by paeoniflorin through the upregulation of urokinase-type plasminogen activator via the MAPK signaling pathway. Molecular Medicine Reports 13:4593-4598

Yeh CF, Chang JS, Wang KC, Shieh DE, Chiang LC (2013) Water extract of Cinnamomum cassia Blume inhibited human respiratory syncytial virus by preventing viral attachment, internalization, and syncytium formation. Journal of Ethnopharmacology 147:321-326

Yeo SSM, Tham FY (2012) Anti-quorum sensing and antimicrobial activities of some traditional Chinese medicinal plants commonly used in South-East Asia. Malaysian Journal of Microbiology 8:11-20

Yeum H-S, Lee Y-C, Kim S-H, Roh S-S, Lee J-C, Seo Y-B (2007) Fritillaria cirrhosa, Anemarrhena asphodeloides, Lee-Mo-Tang and Cyclosporine a Inhibit OvalbuminInduced Eosinophil Accumulation and Th2Mediated Bronchial Hyperresponsiveness in a Murine Model of Asthma. Basic \& Clinical Pharmacology \& Toxicology 100:205-213

Yeung W-F, Chung K-F, Man-Ki Poon M, Yan-Yee Ho F, Zhang S-P, Zhang Z-J, Tat-Chi Ziea E, Wong VT (2012) Chinese herbal medicine for insomnia: A systematic review of randomized controlled trials. Sleep Medicine Reviews 16:497-507

Yeung W-F, Chung K-F, Ng K-Y, Yu Y-M, Tat-Chi Ziea E, Fung-Leung Ng B (2014a) A metaanalysis of the efficacy and safety of 
traditional Chinese medicine formula Ganmai Dazao decoction for depression. Journal of Ethnopharmacology 153:309-317

Yeung W-F, Chung K-F, Ng K-Y, Yu Y-M, Ziea ETC, Ng BF-L (2014b) A systematic review on the efficacy, safety and types of Chinese herbal medicine for depression. Journal of Psychiatric Research 57:165-175

Yoon T, Cheon MS, Lee AY, Lee DY, Moon BC, Chun JM, Choo BK, Kim HK (2010a) Antiinflammatory Activity of Methylene Chloride Fraction From Glehnia littoralis Extract via Suppression of NF-KB and Mitogen-Activated Protein Kinase Activity. Journal of Pharmacological Sciences 112:46-55

Yoon T, Lee DY, Lee AY, Choi G, Choo BK, Kim HK (2010b) Anti-inflammatory effects of Glehnia littoralis extract in acute and chronic cutaneous inflammation. Immunopharmacology and Immunotoxicology 32:663-670

You JS, Lee YJ, Kim KS, Kim SH, Chang KJ (2014) Anti-obesity and hypolipidaemic effects of Nelumbo nucifera seed ethanol extract in human pre-adipocytes and rats fed a high-fat diet. Journal of the Science of Food and Agriculture 94:568-575

Yu L, Shirai N, Suzuki H, Hosono T, Nakajima Y, Kajiwara M, Takatori K (2008) Effect of Lipid Extracted from Tsao-ko (Amomum tsao-ko Crevost et Lemaire) on Digestive Enzyme Activity, Antioxidant Activity, Plasma and Liver Lipids, and Blood Glucose Levels of Mice. Journal of Nutritional Science and Vitaminology 54:378-383

Yu L, Suzuki H (2007) Effects of tsao-ko, Turmeric and Garlic on Body Fat Content and Plasma Lipid Glucose and Liver Lipid Levels in Mice (A comparative Study of Spices). Food Science and Technology Research 13:241-246

Yu TX, Zhang P, Guan Y, Wang M, Zhen MQ (2015) Protective effects of luteolin against cognitive impairment induced by infusion of $A \beta$ peptide in rats. International Journal of Clinical and Experimental Pathology 8:6740-6747

Zada W, Zeeshan S, Bhatti HA, Mahmood W, Rauf K, Abbas G (2016) Cinnamomum cassia : an implication of serotonin reuptake inhibition in animal models of depression. Natural Product Research 30:1212-1214
Zaidi SF, Aziz M, Muhammad JS, Kadowaki M (2015) Diverse pharmacological properties of Cinnamomum cassia: A review. Pakistan Journal of Pharmaceutical Sciences 28:1433-1438

Zare-Zardini H, Tolueinia B, Hashemi A, Ebrahimi L, Fesahat F (2013) Antioxidant and cholinesterase inhibitory activity of a new peptide from Ziziphus jujuba fruits. American Journal of Alzheimer's Disease \& Other Dementias 28:702-709

Zarmouh N, Messeha S, Elshami F, Soliman K (2016) Natural Products Screening for the Identification of Selective Monoamine Oxidase-B Inhibitors. European Journal of Medicinal Plants 15:1-16

Zhan H-D, Zhou H-Y, Sui Y-P, Du X-L, Wang W, Dai L, Sui F, Huo H-R, Jiang T-L (2016) The rhizome of Gastrodia elata Blume - An ethnopharmacological review. Journal of Ethnopharmacology 189:361-385

Zhang L, Yang B, Yu B (2015) Paeoniflorin Protects against Nonalcoholic Fatty Liver Disease Induced by a High-Fat Diet in Mice. Biological \& Pharmaceutical Bulletin 38:1005-1011

Zhang R, Zhou J, Jia Z, Zhang Y, Gu G (2004) Hypoglycemic effect of Rehmannia glutinosa oligosaccharide in hyperglycemic and alloxan-induced diabetic rats and its mechanism. Journal of Ethnopharmacology 90:39-43

Zhang S, He B, Ge J, Li H, Luo X, Zhang H, Li Y, Zhai C, Liu P, Liu X, Fei X (2010) Extraction, chemical analysis of Angelica sinensis polysaccharides and antioxidant activity of the polysaccharides in ischemia-reperfusion rats. International Journal of Biological Macromolecules 47:546-550

Zhang TT, Lu CL, Jiang JG (2014a) Bioactivity evaluation of ingredients identified from the fruits of Amomum tsaoko, a Chinese spice. Food \& Function 5:1747-1754.

Zhang XY, Han XL, Qian ZB (2014b) Study on Optimization of Extraction Process of Saponins from Bulbus Lilii and Their Antidepressant Effects. Asian Journal of Chemistry 26:5970-5972

Zhang Y, Hou G, Yue Y (2000) The analgesic action of Semen Coicis on severe functional dysmenorrhea - a sequential trial observation. 
Journal of traditional Chinese medicine 20:293-296

Zhang $Y$, Sun D, Meng Q, Guo W, Chen $Q$, Zhang $Y$ (2016) Calcium channels contribute to albiflorin-mediated antinociceptive effects in mouse model. Neuroscience Letters 628:105-109

Zhang Z, Wu MD (2006) Protective effect of jujube dates polysaccharide on chemical liver injury in mice and antifatigue effect. Journal of Nanjing Agricultural University 29:94-97

Zhao B, Wu CJ, Gao H, Li X, Zhang WG, Wang H (2008a) Sedative and hypnotic effect of Ophiopogon japonicus on mice. Journal of Xianning College (Medical Sciences) 22:282-284

Zhao WH, Zhao CY, Gao LF, Feng FF, Gao W, Chen ZL, Zhang F, Cao LG, Bi XY, Chen Y, Zhu QY, Zhang YX (2008b) The novel inhibitory effect of Pangdahai on fatty acid synthase. IUBMB Life 60:185-194

Zhaobao W, Bing Y (2010) Gastrodia elata Blume extract ameliorates exercise-induced fatigue. African Journal of Biotechnology 9:5978-5982

Zheng $C$, Dong $Q$, Chen $H$, Cong $Q$, Ding K (2015a) Structural characterization of a polysaccharide from Chrysanthemum morifolium flowers and its antioxidant activity. Carbohydrate Polymers 130:113-121

Zheng C, Dong Q, Du Z, Wang P, Ding K (2015b) Structural elucidation of a polysaccharide from Chrysanthemum morifolium flowers with anti-angiogenic activity. International Journal of Biological Macromolecules 79:674-680

Zheng $\mathrm{H}$, Yang $\mathrm{E}$, Peng $\mathrm{H}$, Li J, Chen S, Zhou J, Fang H, Qu B, Wang Z (2014a) Gastrodin prevents steroid-induced osteonecrosis of the femoral head in rats by anti-apoptosis. Chin Med J (Engl) 127:3926-3931

Zheng Y, Wu Z, Ni H, Ke L, Tong Z, Li W, Li N, Li $\mathrm{J}$ (2014b) Codonopsis pilosula Polysaccharide Attenuates Cecal Ligation and Puncture Sepsis via Circuiting Regulatory T Cells in Mice. Shock 41:250-255

Zhong Y, Deng Y, Chen Y, Chuang PY, He JC (2013) Therapeutic use of traditional Chinese herbal medications for chronic kidney diseases. Kidney International 84:1108-1118

Zhou Y-J, Xiang J-Z, Yuan H, Liu H, Tang Q, Hao H-Z, Yin Z, Wang J, Ming Z-Y (2013) Neferine exerts its antithrombotic effect by inhibiting platelet aggregation and promoting dissociation of platelet aggregates. Thrombosis Research 132:202-210

Zhu W, Xue X, Zhang Z (2016a) Ultrasonicassisted extraction, structure and antitumor activity of polysaccharide from Polygonum multiflorum. International Journal of Biological Macromolecules 91:132-142.

Zhu Y, Wang L, Yang Z, Wang J, Li W, Zhou J, Zhang J (2016b) Hematopoietic effects of Paeoniflorin and Albiflorin on radiotherapyinduced myelosuppression mice. EvidenceBased Complementary and Alternative Medicine 2016:1-8

Zhu YP (1998) Chinese Materia Medica: Chemistry, Pharmacology and Applications. CRC Press, Boca Ratón, FL, USA

Received: 02 November 2016

Accepted: 10 January 2017

Published: 18 February 2017 